\title{
The Synthesis and Evaluation of Amidoximes as Cytotoxic Agents on Model Bacterial E. coli Strains
}

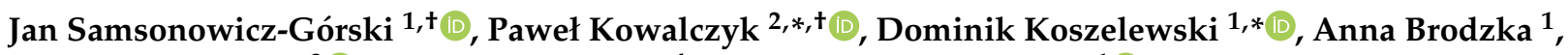 \\ Mateusz Szymczak ${ }^{3}{ }^{(0)}$, Karol Kramkowski ${ }^{4}$ and Ryszard Ostaszewski ${ }^{1}$ (I) \\ 1 Institute of Organic Chemistry PAS, Kasprzaka 44/52, 01-224 Warsaw, Poland; \\ jan.samsonowicz-gorski.stud@pw.edu.pl (J.S.-G.); anna.brodzka@icho.edu.pl (A.B.); \\ ryszard.ostaszewski@icho.edu.pl (R.O.) \\ 2 Department of Animal Nutrition, The Kielanowski Institute of Animal Physiology and Nutrition, \\ Polish Academy of Sciences, Instytucka 3, 05-110 Jabłonna, Poland \\ 3 Department of Molecular Virology, Institute of Microbiology, Faculty of Biology, University of Warsaw, \\ Miecznikowa 1, 02-096 Warsaw, Poland; mszymczak@biol.uw.edu.pl \\ 4 Department of Physical Chemistry, Medical University of Bialystok, Kilińskiego 1 Str., \\ 15-089 Białystok, Poland; kkramk@wp.pl \\ * Correspondence: p.kowalczyk@ifzz.pl (P.K.); dominik.koszelewski@icho.edu.pl (D.K.) \\ + These authos have equally contributed equally to the paper.
}

check for updates

Citation: Samsonowicz-Górski, J.; Kowalczyk, P.; Koszelewski, D.; Brodzka, A.; Szymczak, M.;

Kramkowski, K.; Ostaszewski, R. The Synthesis and Evaluation of Amidoximes as Cytotoxic Agents on Model Bacterial E. coli Strains. Materials 2021, 14, 7577. https:// doi.org/10.3390/ma14247577

Academic Editor: Claudio Canale

Received: 18 November 2021

Accepted: 7 December 2021

Published: 9 December 2021

Publisher's Note: MDPI stays neutral with regard to jurisdictional claims in published maps and institutional affiliations.

Copyright: (c) 2021 by the authors. Licensee MDPI, Basel, Switzerland. This article is an open access article distributed under the terms and conditions of the Creative Commons Attribution (CC BY) license (https:/ / creativecommons.org/licenses/by/ $4.0 /)$.

\begin{abstract}
The biological research on newly synthesized amidoximes, Boc-protected amidoximes and Boc-derived amidines, obtained by a reduction of the parent amidoximes is reported, herein. Due to the presence of a free amino group in both amidines and amidoximes, these compounds can undergo various chemical reactions such as $N$-alkylation and $N$-acylation. One such reaction is Boc-protection, often used in organic synthesis to protect the amino and imino groups. Until now, Boc-protected amidoximes have not been tested for biological activity. Amidoxime derivatives were tested on bacterial E. coli strains. Initial cellular studies tests and digestion with Fpg after the modification of bacterial DNA, suggest that these compounds may have greater potential as antibacterial agents compared to antibiotics such as ciprofloxacin (ci), bleomycin (b) and cloxacillin (cl). The described compounds are highly specific for pathogenic E. coli strains on the basis of the model strains used and may be used in the future as new substitutes for commonly used antibiotics in clinical and hospital infections in the pandemic era.
\end{abstract}

Keywords: amidoximes; Boc-protected amidoximes; Boc-derived amidines; DNA-N-glycosylase; Fpg protein-formamidopyrimidine; lipopolysaccharide (LPS)

\section{Introduction}

Amidoximes perform many activities on various microorganisms and complex eukaryotic organisms. Thus, they are intensively studied as potential drugs, prodrugs or fungicidal or bactericidal substances (Figure 1).<smiles>NC(CSc1nc2cccnc2s1)=NO</smiles><smiles>NC(=O)CC(=O)Nc1ccccc1</smiles>
lysine-specific demethylase inhibitor<smiles></smiles>

Figure 1. Pharmaceutically relevant amidoximes. 
In natural metabolism oximes (derived from arginine) are donors of nitric oxide (NO) [1], which is known to be an important neurotransmitter and neuromodulator and agent targeted in medical treatment [2]. Thus, synthetic amidoximes have impact on natural metabolic pathways on mammalian organisms. Additionally, the cardiotonic activities of amidoximes have been reported [3]. Similar to keto- and aldoximes, amidoximes possess an ability to reactivate aceltylcholinesterase after poisoning with organophosphorus compounds [4]. Furthermore, inhibitory effect of amidoximes on lysine-specific demethylases, important in processes of gene expression, was investigated [5]. Additionally, amidoximes possess documented anti-microbial activity as inhibitors of SHC (squalene-hopene cyclase), which plays a crucial role in the process of stabilization of cell membrane (for instance in Alicyclobacillus acidocaldarius cells) [6]. Thus, amidoximes were examined for use in surface functionalization of nanofibers [7] or material utilized for adsorption of uranium from seawater [8]. Following anti-microorganism activity, anti-virial activity on influenza B-Mass virus [9] of pyridine amidoxime derivative was also proven. Amidoximes are reduced in vivo to corresponding amidines by organisms possessing active ARC (amidoxime reducing component). Such process may result in release of substances exhibiting anti-microbial activity. Drugs against pneumocystis, protozoan [1] or trypanosoma may be introduced this way [10]. Furthermore, reports have been made on amidine-containing inhibitors of serine proteases, which also enlarge their utility in medicine [11].

Analyzed K12 and R2-R4 strains of Escherichia coli (E. coli) are not only the dominant species of the human aerobic bacterial flora and are flora in various habitats in which people live, e.g., bathrooms, clinics and hospitals, but they can temporarily colonize the oropharynx and skin in healthy people. However, apart from saprophytic strains, harmless to humans, there are also strains of Escherichia coli that are pathogenic for humans and cause various forms of acute diarrhea. Infection usually occurs through contaminated food and water, as is the case with other bacterial diarrhea, and less frequently through indirect contact. In industrialized countries, pathogenic enteric strains of Escherichia coli are rarely a component of the intestinal flora of healthy people, therefore they are considered strictly pathogenic bacteria. When an appropriate volume of bacteria is ingested by a person susceptible to pathogenic infection, strains of Escherichia coli have the ability to cause inflammation of the small intestine and/or large intestine. Adequate gastric acidity has a disinfecting effect and protects to a certain extent against infection, therefore people with low gastric acidity are particularly susceptible to infections with pathogenic Escherichia coli strains. The source of the infection is a sick person or a vector (except for STEC/EHEC strains-the source is cattle). Intestinal diseases caused by pathogenic strains of Escherichia coli occur in the form of epidemics or as sporadic cases, with an increase in the incidence in the summer months, which is the rule for bacterial diarrhea. Therefore, we study these strains with the use of the analyzed amidoximes to investigate their etiology and the mechanism causing their resistance to many known and commonly used antibiotics [11-48].

Despite numbers of reports in the literature on amidoxime's pharmacological and biological properties, their anti-bacterial activities are still being rediscovered, therefore there is still a need for additional research on their cytotoxic effect on selected hospital bacterial strains causing diseases associated with blood infections such as sepsis. Therefore, this article is an attempt to address this question. Until now, such compounds have not been tested for biological activity against pathogenic E. coli strains, so there is a need to clarify their role.

\section{Materials and Methods}

All compounds for the research were obtained from Sigma-Aldrich (Merck Life Science LTD division in Poznań, Poland) 


\section{Experimental Section}

\subsection{2-(4-Methoxybenzylamino)-2-oxo-1-(4-cyanophenyl)ethyl phenylacetate}

p-Methoxybenzylisocyanide (147.2 $\mathrm{mg}, 1 \mathrm{mmol}$ ) was added to a suspension of 4cyanobenzaldehyde (131.1, $1 \mathrm{mmol})$ and phenylacetic acid $(136.2 \mathrm{mg}, 1 \mathrm{mmol})$ at room temperature. The reaction mixture was stirred at ambient temperature for $18 \mathrm{~h}$. The crude product was purified by column chromatography with silica gel (hexanes/EtOAc) to afford the corresponding product as a colorless semi-solid in $72 \%$ yield $(298 \mathrm{mg}, 0.72 \mathrm{mmol})$. $\mathrm{R}_{\mathrm{f}} 0.28$ (hexanes/EtOAc, 7:3). ${ }^{1} \mathrm{H}$ NMR $\left(400 \mathrm{MHz}, \mathrm{CDCl}_{3}\right) \delta 7.63-7.53(\mathrm{~m}, 2 \mathrm{H}), 7.44(\mathrm{~d}$, $J=8.1 \mathrm{~Hz}, 2 \mathrm{H}), 7.31-7.17(\mathrm{~m}, 5 \mathrm{H}), 7.10-6.97(\mathrm{~m}, 2 \mathrm{H}), 6.89-6.77(\mathrm{~m}, 2 \mathrm{H}), 6.32(\mathrm{~s}, 1 \mathrm{H}), 6.10$ $(\mathrm{s}, 1 \mathrm{H}), 4.22(\mathrm{~d}, J=5.7 \mathrm{~Hz}, 2 \mathrm{H}), 3.79(\mathrm{~s}, 3 \mathrm{H}), 3.72(\mathrm{~s}, 2 \mathrm{H}) ;{ }^{13} \mathrm{C} \mathrm{NMR}\left(100 \mathrm{MHz}, \mathrm{CDCl}_{3}\right) \delta$ 169.3, 167.1, 159.1, 140.5, 133.0, 132.3, 129.4, 129.1, 129.1, 128.9, 128.95 128.8, 128.8, 127.7, $127.5,127.5,118.3,114.1,112.5,60.3,42.8,41.1,20.9$. Elemental analysis calculated for $\mathrm{C}_{25} \mathrm{H}_{22} \mathrm{~N}_{2} \mathrm{O}_{4}$ : C, $72.45 \%$; $\mathrm{H}, 5.35 \%$; N, 6.76\%; found: $\mathrm{C}, 72.29 \%$; $\mathrm{H}, 5.31 \%$; N, $6.68 \%$

\subsection{4-Cyanostilbene}

The $\mathrm{PdCl}_{2}$ catalyst $(0.02 \mathrm{~g}, 1 \mathrm{~mol} \%)$ was added to a mixture of 4-bromobenzenecarbonitrile (182 mg, $1 \mathrm{mmol})$, trans-2-phenylvinylboronic acid (177 mg, $1.2 \mathrm{mmol})$, and $\mathrm{K}_{2} \mathrm{CO}_{3}(2 \mathrm{mmol})$ in $10 \mathrm{~mL}$ of EtOH with $1 \mathrm{~mL}$ of $\mathrm{H}_{2} \mathrm{O}$, and stirred at room temperature. The reaction was followed by TLC. After the completion of the reaction, the mixture was washed with diethyl ether $(3 \times 10 \mathrm{~mL})$ followed by deionized water $(3 \times 10 \mathrm{~mL})$. The organic phase after separation of the catalyst was evaporated to obtain the crude product. The product was purified by column chromatography with silica gel (hexanes/EtOAc) to afford the corresponding product as a colorless solid in $82 \%$ yield $(168 \mathrm{mg}, 0.82 \mathrm{mmol}) ; \mathrm{m} . \mathrm{p} .117^{\circ} \mathrm{C}$, (Lit. 116-118 $\left.{ }^{\circ} \mathrm{C}\right)[12] ;{ }^{1} \mathrm{H}$ NMR $\left(400 \mathrm{MHz}, \mathrm{CDCl}_{3}\right)$ 8 7.66-7.59 (m, 2H), 7.59-7.50 (m, 4H), 7.43-7.36 (m, 2H), 7.36-7.28 (m, 1H), 7.21 (d, J = 16.3 Hz, 1H), $7.08(\mathrm{~d}, J=16.4 \mathrm{~Hz}, 1 \mathrm{H})$; ${ }^{13} \mathrm{C}$ NMR $\left(100 \mathrm{MHz}, \mathrm{CDCl}_{3}\right) \delta 141.8,136.3,132.4,128.8,128.6,126.9,119.0,110.6 .{ }^{1} \mathrm{H}-$ and ${ }^{13} \mathrm{CNMR}$ data were in accordance with those reported in the literature [13].

\subsection{4-Methoxybiphenyl-4-carbonitrile}

A mixture of 4-bromobenzenecarbonitrile ( $182 \mathrm{mg}, 1 \mathrm{mmol})$, 4-methoxyphenylboronic acid (152 mg, $1 \mathrm{mmol}), \mathrm{PdCl}_{2}(0.0025 \mathrm{mmol}, 0.44 \mathrm{mg}), \mathrm{K}_{2} \mathrm{CO}_{3}(1 \mathrm{mmol})$ was stirred in distilled water $(3 \mathrm{~mL})$ and ethanol $(3 \mathrm{~mL})$ at room temperature. The reaction was followed by TLC. After the completion of the reaction, the mixture was quenched by brine $(15 \mathrm{~mL})$, extracted with diethyl ether $(4 \times 10 \mathrm{~mL})$, dried by anhydrous $\mathrm{MgSO}_{4}$, concentrated under vacuum. The crude product was purified by column chromatography with silica gel (hexanes/EtOAc) to afford the corresponding product as a colorless solid in $95 \%$ yield (198 mg, 0.95 mmol); m.p. $102{ }^{\circ} \mathrm{C}$ (Lit. m.p. $\left.101-103{ }^{\circ} \mathrm{C}\right)[14] ;{ }^{1} \mathrm{H}$ NMR $\left(400 \mathrm{MHz}, \mathrm{CDCl}_{3}\right) \delta$ $7.61(\mathrm{q}, J=8.7 \mathrm{~Hz}, 4 \mathrm{H}), 7.51(\mathrm{~d}, J=8.8 \mathrm{~Hz}, 2 \mathrm{H}), 6.98(\mathrm{~d}, J=8.8 \mathrm{~Hz}, 2 \mathrm{H}), 3.83(\mathrm{~s}, 3 \mathrm{H}) .{ }^{1} \mathrm{HNMR}$ data were in accordance with those reported in the literature [15].

\subsection{General Procedure for Synthesis of Amidoximes 1-19}

A solution of nitrile $(2.5 \mathrm{mmol})$, hydroxylamine hydrochloride $(695 \mathrm{mg}, 10.0 \mathrm{~mol})$, sodium carbonate $(530 \mathrm{mg}, 5.0 \mathrm{~mol})$, water $(6 \mathrm{~mL})$ and ethanol $(9 \mathrm{~mL})$ was refluxed for $3 \mathrm{~h}$. The reaction was allowed to cool, and the ethanol was removed under reduced pressure. The aqueous layer was extracted with ethyl acetate $(3 \times 10 \mathrm{~mL})$; the combined organic fractions were dried over anhydrous $\mathrm{Na}_{2} \mathrm{SO}_{4}$ and the solvent removed under reduced pressure to afford the desired amidoximes $1-19$, which were sufficiently pure for use without further purification (Scheme 1, Figure 2). 


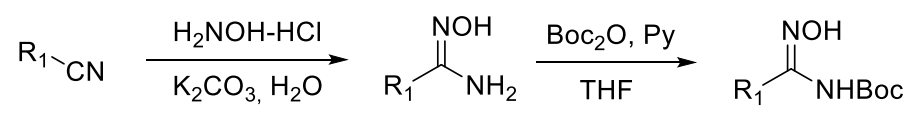

$$
\begin{aligned}
& \begin{array}{lll}
\mathrm{R}_{1} \text { :Alkyl, Aryl } & \mathbf{1 - 1 9} & \mathbf{2 0 - 2 3}
\end{array} \\
& \text { 1. } \mathrm{H}_{2} / \mathrm{Pd} \\
& \text { 2. } \mathrm{Boc}_{2} \mathrm{O} \\
& \overbrace{\text { NHBoc }}^{\mathrm{NH}} \\
& \text { 24-26 }
\end{aligned}
$$

Scheme 1. Synthesis of the studied compounds 1-26.

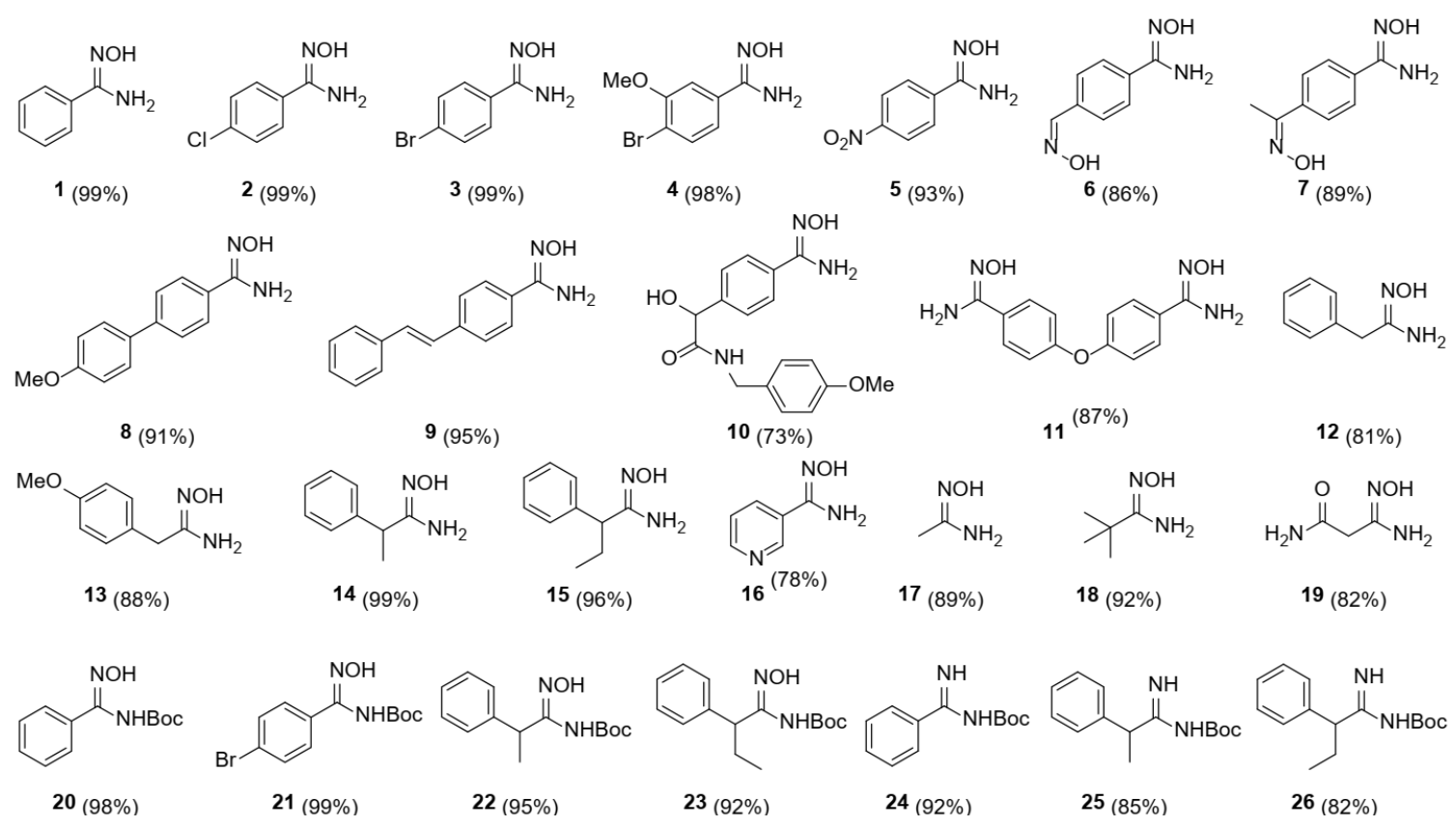

Figure 2. The chemical structures of the obtained amidine derivatives 1-26. Yields are provided in brackets.

\subsubsection{N'-Hydroxybenzimidamide (1)}

Compound 1 was obtained according to General method with $99 \%$ yield (340 mg, $2.5 \mathrm{mmol}$ ) as white crystals; m.p. 70-71 ${ }^{\circ} \mathrm{C}$ (Lit. m.p. 67-69 $\left.{ }^{\circ} \mathrm{C}\right)$ [16] ${ }^{1} \mathrm{H}$ NMR $(400 \mathrm{MHz}$, DMSO-d 6 ) $\delta 9.59(\mathrm{~s}, 1 \mathrm{H}), 7.73-7.58(\mathrm{~m}, 2 \mathrm{H}), 7.41-7.22(\mathrm{~m}, 3 \mathrm{H}), 5.75(\mathrm{~s}, 2 \mathrm{H}) ;{ }^{13} \mathrm{C} \mathrm{NMR}$ $\left(100 \mathrm{MHz}, \mathrm{DMSO}-\mathrm{d}_{6}\right) \delta 151.3,133.8,129.3,128.5,125.8 .{ }^{1} \mathrm{H}$ and ${ }^{13} \mathrm{C}$ NMR data were in accordance with those reported in the literature [17].

\subsubsection{4-Chlorobenzamidoxime (2)}

Compound 2 was obtained according to General method with $99 \%$ yield (422 mg, $2.5 \mathrm{mmol}$ ) as white crystals m.p. $130-131^{\circ} \mathrm{C}$ (Lit. m.p. $\left.128-130{ }^{\circ} \mathrm{C}\right)$ [18]; ${ }^{1} \mathrm{H} \mathrm{NMR}(400 \mathrm{MHz}$, DMSO-d $\left.\mathrm{d}_{6}\right) \delta 9.70(\mathrm{~s}, 1 \mathrm{H}), 7.68(\mathrm{~d}, J=8.7 \mathrm{~Hz}, 2 \mathrm{H}), 7.41(\mathrm{~d}, J=8.6 \mathrm{~Hz}, 2 \mathrm{H}), 5.83(\mathrm{~s}, 2 \mathrm{H}) ;{ }^{13} \mathrm{C}$ NMR $\left(100 \mathrm{MHz}, \mathrm{DMSO}-\mathrm{d}_{6}\right) \delta 150.4,133.9,128.5,127.5 .{ }^{1} \mathrm{H}$ and ${ }^{13} \mathrm{C}$ NMR data were in accordance with those reported in the literature [19].

\subsection{3. p-Bromobenzamidoxime (3)}

Compound 3 was obtained according to General method with $99 \%$ yield (372 mg, $2.5 \mathrm{mmol}$ ) as white crystals; m.p. $142-143{ }^{\circ} \mathrm{C}$; (Lit. m.p. $140-141{ }^{\circ} \mathrm{C}$ ) [20]; ${ }^{1} \mathrm{H}$ NMR $\left(400 \mathrm{MHz}, \mathrm{DMSO}_{-} \mathrm{d}_{6}\right) \delta 9.72(\mathrm{~s}, 1 \mathrm{H}), 7.86-7.31(\mathrm{~m}, 4 \mathrm{H}), 5.82(\mathrm{~s}, 2 \mathrm{H}) ;{ }^{13} \mathrm{C} \mathrm{NMR}(100 \mathrm{MHz}$, DMSO-d $\left._{6}\right) \delta 150.5,133.0,131.5,127.8,122.5 .{ }^{1} \mathrm{H}$ and ${ }^{13} \mathrm{C}$ NMR data were in accordance with those reported in the literature [21]. 


\subsubsection{4-Bromo-N'-hydroxy-3-methoxybenzimidamide (4)}

Compound 4 was obtained according to General method with $98 \%$ yield $(600 \mathrm{mg}$, $2.4 \mathrm{mmol})$ as white crystals, m.p. $128-129{ }^{\circ} \mathrm{C} ;{ }^{1} \mathrm{H}$ NMR $\left(400 \mathrm{MHz}, \mathrm{DMSO}-\mathrm{d}_{6}\right) \delta 9.69(\mathrm{~s}$, $1 \mathrm{H}), 7.53(\mathrm{~d}, J=8.3 \mathrm{~Hz}, 1 \mathrm{H}), 7.34(\mathrm{~d}, J=1.9 \mathrm{~Hz}, 1 \mathrm{H}), 7.19(\mathrm{dd}, J=8.3,1.9 \mathrm{~Hz}, 1 \mathrm{H}), 5.86(\mathrm{~s}$, $2 \mathrm{H}), 3.85(\mathrm{~s}, 3 \mathrm{H}) ;{ }^{13} \mathrm{C}$ NMR $\left(100 \mathrm{MHz}, \mathrm{DMSO}_{6}\right) \delta 155.5,150.5,134.7,132.9,119.3,111.5$, 109.9, 56.6. Elemental analysis calculated for $\mathrm{C}_{8} \mathrm{H}_{9} \mathrm{BrN}_{2} \mathrm{O}_{2}: \mathrm{C}, 39.21 \% ; \mathrm{H}, 3.70 \% ; \mathrm{N}, 11.43 \%$; found: $\mathrm{C}, 39.18 \% ; \mathrm{H}, 3.65 \%$; N, $11.37 \%$.

\subsubsection{N'-Hydroxy-4-nitrobenzimidamide (5)}

Compound 5 was obtained according to General method with $93 \%$ yield (421 mg, $2.3 \mathrm{mmol}$ ) as white crystals; m.p. $185^{\circ} \mathrm{C}$ (Lit. m.p. $\left.188-190{ }^{\circ} \mathrm{C}\right)$ [21]; ${ }^{1} \mathrm{H}$ NMR $(400 \mathrm{MHz}$, DMSO-d $\left._{6}\right) \delta 8.98(\mathrm{~s}, 1 \mathrm{H}), 8.13(\mathrm{~d}, J=8.7 \mathrm{~Hz}, 2 \mathrm{H}), 7.52(\mathrm{~d}, J=8.7 \mathrm{~Hz}, 2 \mathrm{H}), 5.50(\mathrm{~s}, 2 \mathrm{H}) ;{ }^{13} \mathrm{C}$ NMR (100 MHz, DMSO-d 6 ) $\delta 151.5,146.7,130.4,123.6 .{ }^{1} \mathrm{H}$ and ${ }^{13} \mathrm{C}$ NMR data were in accordance with those reported in the literature [22]

\subsubsection{N'-Hydroxy-4-((hydroxyimino)methyl)benzimidamide (6)}

Compound 6 was obtained according to General method with $86 \%$ yield $(385 \mathrm{mg}$, $2.1 \mathrm{mmol})$ as white crystals; m.p. $211-213{ }^{\circ} \mathrm{C} ;{ }^{1} \mathrm{H}$ NMR $\left(400 \mathrm{MHz}, \mathrm{DMSO}-\mathrm{d}_{6}\right) \delta 11.25(\mathrm{~s}$, $1 \mathrm{H}), 9.68(\mathrm{~s}, 1 \mathrm{H}), 8.12(\mathrm{~s}, 1 \mathrm{H}), 7.68(\mathrm{~d}, J=8.4 \mathrm{~Hz}, 2 \mathrm{H}), 7.56(\mathrm{~d}, J=8.4 \mathrm{~Hz}, 2 \mathrm{H}), 5.79(\mathrm{~s}, 2 \mathrm{H})$; ${ }^{13} \mathrm{C}$ NMR $\left(100 \mathrm{MHz}\right.$, DMSO-d 6 ) $\delta 150.8,148.2,134.4,133.9,126.5,126.0 .{ }^{1} \mathrm{H}$ and ${ }^{13} \mathrm{C}$ NMR data were in accordance with those reported in the literature [23].

\subsubsection{N'-Hydroxy-4- [N-hydroxyethanimidoyl]benzene-1-carboximidamide (7)}

Compound 7 was obtained according to General method with $89 \%$ yield $(430 \mathrm{mg}$, $2.2 \mathrm{mmol}$ ) as white crystals; m.p. $192-193{ }^{\circ} \mathrm{C} ;{ }^{1} \mathrm{H}$ NMR $\left(400 \mathrm{MHz}, \mathrm{DMSO}-\mathrm{d}_{6}\right) \delta 11.20(\mathrm{~s}$, $1 \mathrm{H}), 9.65(\mathrm{~s}, 1 \mathrm{H}), 7.71-7.59(\mathrm{~m}, 4 \mathrm{H}), 5.78(\mathrm{~s}, 2 \mathrm{H}), 2.14(\mathrm{~s}, 3 \mathrm{H}) ;{ }^{13} \mathrm{C} \mathrm{NMR}\left(100 \mathrm{MHz}, \mathrm{DMSO}-\mathrm{d}_{6}\right)$ $\delta$ 153.0, 150.9, 137.7, 125.7, 125.6, 11.8. ${ }^{1} \mathrm{H}$ and ${ }^{13} \mathrm{C}$ NMR data were in accordance with those reported in the literature [24].

\subsubsection{4-(4-Methoxyphenyl)phenyl]-nitrosomethanamine (8)}

Compound 8 was obtained according to General method with $91 \%$ yield $(551 \mathrm{mg}$, $2.3 \mathrm{mmol})$ as white crystals; m.p. $143-145{ }^{\circ} \mathrm{C}^{1}{ }^{1} \mathrm{H}$ NMR (400 MHz, DMSO-d6) $\delta 9.65(\mathrm{~s}$, 1H), 7.73-7.58 (m, 7H), 7.01-6.98 (m, 2H), $5.78(\mathrm{~s}, 2 \mathrm{H}), 3.76(\mathrm{~s}, 3 \mathrm{H}) ;{ }^{13} \mathrm{C}$ NMR $(100 \mathrm{MHz}$, DMSO-d6) $\delta 159.5,151.1,132.2,132.0,128.6,128.4,128.1,126.3,126.2,126.2,114.9,114.8$, 55.6. HR-MS (ESI) $(\mathrm{M}+\mathrm{H})^{+} \mathrm{m} / \mathrm{z}$ calculated for $\mathrm{C}_{14} \mathrm{H}_{15} \mathrm{~N}_{2} \mathrm{O}_{2}$ : 243.1134; found: 243.1132 . Elemental analysis calculated for $\mathrm{C}_{14} \mathrm{H}_{14} \mathrm{~N}_{2} \mathrm{O} 2$ : $\mathrm{C}, 69.41 \% ; \mathrm{H}, 5.82 \%$;, $11.56 \%$; found: $\mathrm{C}$, $69.24 \% ; \mathrm{H}, 5.91 \%$; N, $11.38 \%$.

\subsubsection{N'-Hydroxy-4- [(E)-2-phenylethenyl]benzene-1-carboximidamide (9)}

Compound 9 was obtained according to General method with $95 \%$ yield $(566 \mathrm{mg}$, $2.3 \mathrm{mmol})$ as white crystals, m.p. $133-134{ }^{\circ} \mathrm{C} ;{ }^{1} \mathrm{H}$ NMR $\left(400 \mathrm{MHz}, \mathrm{DMSO}-\mathrm{d}_{6}\right) \delta 9.63(\mathrm{~s}$, $1 \mathrm{H}), 7.77-7.53(\mathrm{~m}, 6 \mathrm{H}), 7.36(\mathrm{t}, J=7.6 \mathrm{~Hz}, 2 \mathrm{H}), 7.32-7.14(\mathrm{~m}, 3 \mathrm{H}), 5.77(\mathrm{~s}, 2 \mathrm{H}) ;{ }^{13} \mathrm{C}$ NMR $\left(100 \mathrm{MHz}, \mathrm{DMSO}_{\mathrm{d}}\right) \delta$ 151.0, 138.0, 137.4, 132.8, 129.1, 128.3, 126.9, 126.6, 126.0, 117.4, 109.0. Elemental analysis calculated for $\mathrm{C}_{15} \mathrm{H}_{14} \mathrm{~N}_{2} \mathrm{O}: \mathrm{C}, 75.61 \% ; \mathrm{H}, 5.92 \% ; \mathrm{N}, 11.76 \%$; found: C, $75.55 \% ; \mathrm{H}, 5.82 \%$; N, $11.64 \%$.

3.4.10. 2-\{4-[1-Amino-2-hydroxyethenyl]phenyl\}-2-hydroxy-N-[(4methoxyphenyl)methyl]acetamide (10)

Compound 10 was obtained according to General method with 73\% yield (601 mg, $1.8 \mathrm{mmol})$ as white crystals m.p. $151-152{ }^{\circ} \mathrm{C} ;{ }^{1} \mathrm{H}$ NMR $\left(400 \mathrm{MHz}, \mathrm{DMSO}-\mathrm{d}_{6}\right) \delta 8.49(\mathrm{~s}$, $1 \mathrm{H}), 7.78(\mathrm{~d}, J=8.0 \mathrm{~Hz}, 2 \mathrm{H}), 7.60(\mathrm{~d}, J=8.2 \mathrm{~Hz}, 2 \mathrm{H}), 7.10(\mathrm{~d}, J=8.0 \mathrm{~Hz}, 2 \mathrm{H}), 6.82(\mathrm{~d}$, $J=8.1 \mathrm{~Hz}, 2 \mathrm{H}), 6.42(\mathrm{~s}, 1 \mathrm{H}), 5.05(\mathrm{~s}, 1 \mathrm{H}), 4.17(\mathrm{~s}, 2 \mathrm{H}), 3.69(\mathrm{~s}, 3 \mathrm{H})$. HR-MS (ESI) $(\mathrm{M}+\mathrm{H})^{+}$ $\mathrm{m} / \mathrm{z}$ calculated for $\mathrm{C}_{17} \mathrm{H}_{20} \mathrm{~N}_{3} \mathrm{O}_{4}$ : 329.1495; found: 329.1491. Elemental analysis calculated for $\mathrm{C}_{17} \mathrm{H}_{19} \mathrm{~N}_{3} \mathrm{O}_{4}$ : C, $62.00 \%$; H, 5.81\%; N, $12.76 \%$; found: $\mathrm{C}, 61.89 \%$; $\mathrm{H}, 5.97 \%$; N, $12.54 \%$. 


\subsubsection{4,4'-Oxy-bis-benzamide oxime (11)}

Compound 11 was obtained according to General method with $87 \%$ yield (623 mg, $2.2 \mathrm{mmol}$ ) as white crystals; m.p. $194-195{ }^{\circ} \mathrm{C}$ (Lit. m.p. 193-194 ${ }^{\circ} \mathrm{C}$ ) [25]; ${ }^{1} \mathrm{H}$ NMR $(400 \mathrm{MHz}$, DMSO-d 6$) \delta 7.66(\mathrm{~d}, J=8.2 \mathrm{~Hz}, 4 \mathrm{H}), 6.99(\mathrm{~d}, J=8.2 \mathrm{~Hz}, 4 \mathrm{H}), 5.74(\mathrm{~s}, 4 \mathrm{H}) ;{ }^{13} \mathrm{C}$ NMR (100 MHz, DMSO-d 6 ) $\delta 157.4,151.0,130.2,129.1,127.8,127.7,119.3,118.8,118.7,118.1$. Elemental analysis calculated for $\mathrm{C}_{14} \mathrm{H}_{14} \mathrm{~N}_{4} \mathrm{O}_{3}: \mathrm{C}, 58.74 \% ; \mathrm{H}, 4.93 \%$;, $19.57 \%$; found: $\mathrm{C}$, $58.66 \%$; H, $4.88 \%$; N, $19.45 \%$.

\subsubsection{Benzylamidoxime (12)}

Compound 12 was obtained according to General method with $81 \%$ yield (304 mg, $2.0 \mathrm{mmol}$ ) as white crystals; m.p. $65-66{ }^{\circ} \mathrm{C}$ (Lit. 67-68 $\left.{ }^{\circ} \mathrm{C}\right)$ [26]; ${ }^{1} \mathrm{H}$ NMR $(400 \mathrm{MHz}$, DMSO-d $\left.\mathrm{d}_{6}\right) \delta 8.85(\mathrm{~s}, 1 \mathrm{H}), 7.30-7.11(\mathrm{~m}, 5 \mathrm{H}), 5.33(\mathrm{~s}, 2 \mathrm{H}), 3.25(\mathrm{~s}, 2 \mathrm{H}) ;{ }^{13} \mathrm{C} \mathrm{NMR}(100 \mathrm{MHz}$, DMSO- $\left._{6}\right) \delta 152.3,138.4,129.1,128.5,126.6,37.6 .{ }^{1} \mathrm{H}$ and ${ }^{13} \mathrm{C}$ NMR data were in accordance with those reported in the literature [27].

\subsubsection{N'-Hydroxy-2-(4-methoxyphenyl)ethanimidamide (13)}

Compound 13 was obtained according to General method with $88 \%$ yield (396 mg, $2.2 \mathrm{mmol}$ ) as white crystals; m.p. $111-112{ }^{\circ} \mathrm{C}$ (Lit. m.p. $\left.110-111^{\circ} \mathrm{C}\right)$ [28]; ${ }^{1} \mathrm{H}$ NMR $(400 \mathrm{MHz}$, DMSO-d $\left.\mathrm{d}_{6}\right) \delta 8.80(\mathrm{~s}, 1 \mathrm{H}), 7.16(\mathrm{~d}, J=8.8 \mathrm{~Hz}, 2 \mathrm{H}), 6.82(\mathrm{~d}, J=8.6 \mathrm{~Hz}, 2 \mathrm{H}), 5.27(\mathrm{~s}, 2 \mathrm{H}), 3.70$ $(\mathrm{s}, 3 \mathrm{H}), 3.16(\mathrm{~s}, 2 \mathrm{H}) ;{ }^{13} \mathrm{C}$ NMR $\left(100 \mathrm{MHz}, \mathrm{DMSO}_{\mathrm{d}}\right.$ ) $\delta 158.2,152.7,130.3,130.1,114.0,55.4$, 36.7. ${ }^{1} \mathrm{H}$ and ${ }^{13} \mathrm{C}$ NMR data were in accordance with those reported in the literature [29].

\subsubsection{N'-Hydroxy-2-phenylpropanimidamide (14)}

Compound 14 was obtained according to General method with $99 \%$ yield (406 mg, $2.5 \mathrm{mmol})$ as white crystals; m.p. $138-139{ }^{\circ} \mathrm{C} ;{ }^{1} \mathrm{H}$ NMR $\left(400 \mathrm{MHz}, \mathrm{CDCl}_{3}\right) \delta 7.40-7.24$ $(\mathrm{m}, 5 \mathrm{H}), 6.32(\mathrm{~s}, 1 \mathrm{H}), 4.52(\mathrm{~s}, 2 \mathrm{H}), 3.67(\mathrm{q}, J=7.2 \mathrm{~Hz}, 1 \mathrm{H}), 1.51(\mathrm{~d}, J=7.2 \mathrm{~Hz}, 3 \mathrm{H}) ;{ }^{13} \mathrm{C}$ NMR $\left(100 \mathrm{MHz}, \mathrm{CDCl}_{3}\right) \delta 141.3,128.8,127.3,41.6,18.0$. Elemental analysis calculated for $\mathrm{C}_{9} \mathrm{H}_{12} \mathrm{~N}_{2} \mathrm{O}: \mathrm{C}, 65.83 \% ; \mathrm{H}, 7.37 \%$; N, 17.06\%; found: $\mathrm{C}, 65.77 \% ; \mathrm{H}, 7.32 \% ; \mathrm{N}, 16.98 \%$.

\subsubsection{N-Hydroxy-2-phenyl-butyrimidamide (15)}

Compound 15 obtained according to General method with $96 \%$ yield $(428 \mathrm{mg}, 2.3 \mathrm{mmol}$ ) as white crystals $67-68{ }^{\circ} \mathrm{C} ;{ }^{1} \mathrm{H}$ NMR $\left(400 \mathrm{MHz}, \mathrm{CDCl}_{3}\right) \delta 7.35-7.24(\mathrm{~m}, 5 \mathrm{H}), 4.42(\mathrm{~s}, 2 \mathrm{H})$, $3.32(\mathrm{t}, J=7.7 \mathrm{~Hz}, 1 \mathrm{H}), 2.07-1.80(\mathrm{~m}, 2 \mathrm{H}), 0.91(\mathrm{t}, J=7.3 \mathrm{~Hz}, 3 \mathrm{H}) ;{ }^{13} \mathrm{C} \mathrm{NMR}(100 \mathrm{MHz}$, $\left.\mathrm{CDCl}_{3}\right) \delta 155.2,140.4,128.7,127.8,127.1,49.5,24.8,12.1$. Elemental analysis calculated for $\mathrm{C}_{10} \mathrm{H}_{14} \mathrm{~N}_{2} \mathrm{O}$ : C, $67.39 \% ; \mathrm{H}, 7.92 \%$; N, $15.72 \%$; found: $\mathrm{C}, 67.28 \% ; \mathrm{H}, 7.84 \%$;, $15.55 \%$.

\subsubsection{Nicotinamide oxime (16)}

Compound 16 was obtained according to General method with $78 \%$ yield (267 mg, $1.9 \mathrm{mmol})$ as white crystals; m.p. $108-110{ }^{\circ} \mathrm{C}$ (Lit. 107-109 $\left.{ }^{\circ} \mathrm{C}\right)$ [30]; ${ }^{1} \mathrm{H}$ NMR $(400 \mathrm{MHz}$, DMSO-d 6 ) $\delta 9.80(\mathrm{~s}, 1 \mathrm{H}), 8.84(\mathrm{dd}, J=2.3,1.0 \mathrm{~Hz}, 1 \mathrm{H}), 8.54(\mathrm{dd}, J=4.8,1.7 \mathrm{~Hz}, 1 \mathrm{H}), 7.99$ $(\mathrm{d}, J=8.0 \mathrm{~Hz}, 1 \mathrm{H}), 7.55-7.19(\mathrm{~m}, 1 \mathrm{H}), 5.94(\mathrm{~s}, 2 \mathrm{H}) ;{ }^{13} \mathrm{C}$ NMR (100 MHz, DMSO-d 6 ) $\delta 150.2$, 149.4, 147.0, 133.2, 129.5, 123.6. ${ }^{1} \mathrm{H}$ and ${ }^{13} \mathrm{C}$ NMR data were in accordance with those reported in the literature [31].

\subsubsection{Acetamide oxime (17)}

Compound 17 was obtained according to General method with $89 \%$ yield (165 mg, $2.2 \mathrm{mmol}$ ) as white crystals; m.p. $135-136^{\circ} \mathrm{C}$ (Lit. m.p. $\left.136-137^{\circ} \mathrm{C}\right)$ [32]; ${ }^{1} \mathrm{H}$ NMR $(400 \mathrm{MHz}$, DMSO-d $\left._{6}\right) \delta 8.64(\mathrm{~s}, 1 \mathrm{H}), 5.37(\mathrm{~s}, 2 \mathrm{H}), 1.61(\mathrm{~s}, 3 \mathrm{H}) ;{ }^{13} \mathrm{C} \mathrm{NMR}(100 \mathrm{MHz}$, DMSO-d 6 ) $\delta 150.3$, 17.0. ${ }^{1} \mathrm{H}$ and ${ }^{13} \mathrm{C}$ NMR data were in accordance with those reported in the literature [33].

\subsubsection{N'-Hydroxypivalimidamide (18)}

Compound 18 was obtained according to General method with $92 \%$ yield ( $267 \mathrm{mg}$, $2.3 \mathrm{mmol}$ ) as white crystals, m.p. $112-113^{\circ} \mathrm{C}$ (Lit. m.p. $\left.115-116^{\circ} \mathrm{C}\right)$ [34]; ${ }^{1} \mathrm{H}$ NMR $(400 \mathrm{MHz}$, 
$\left.\mathrm{CDCl}_{3}\right) \delta 8.47(\mathrm{~s}, 1 \mathrm{H}), 4.57(\mathrm{~s}, 2 \mathrm{H}), 1.17(\mathrm{~s}, 9 \mathrm{H}) ;{ }^{13} \mathrm{C} \mathrm{NMR}\left(100 \mathrm{MHz}, \mathrm{CDCl}_{3}\right) \delta$ 159.6, 34.5, 27.8. ${ }^{1} \mathrm{H}$ and ${ }^{13} \mathrm{C}$ NMR data were in accordance with those reported in the literature [35].

\subsubsection{9. $\beta$-Amino- $\beta$-oximinopropioamide (19)}

Compound 19 was obtained according to General method with $82 \%$ yield $(240 \mathrm{mg}$, $2.0 \mathrm{mmol}$ ) as white crystal; m.p. $154-155{ }^{\circ} \mathrm{C}$ (Lit. m.p. $\left.159{ }^{\circ} \mathrm{C}\right)[36] ;{ }^{1} \mathrm{H}$ NMR $(400 \mathrm{MHz}$, $\left.\mathrm{DMSO}_{-} \mathrm{d}_{6}\right) \delta 8.94(\mathrm{~s}, 1 \mathrm{H}), 7.32(\mathrm{~s}, 1 \mathrm{H}), 6.93(\mathrm{~s}, 1 \mathrm{H}), 5.36(\mathrm{~s}, 2 \mathrm{H}), 2.78(\mathrm{~s}, 2 \mathrm{H}) ;{ }^{13} \mathrm{C} \mathrm{NMR}$ $\left(100 \mathrm{MHz}, \mathrm{DMSO}-\mathrm{d}_{6}\right) \delta 149.4,38.3 .{ }^{1} \mathrm{H}$ and ${ }^{13} \mathrm{C}$ NMR data were in accordance with those reported in the literature [37].

\subsection{General Procedure for Synthesis of Compounds 20-23}

Amidoxime ( $1 \mathrm{mmol})$ was dissolved in a mixture of deionized water $(20 \mathrm{~mL}), 2 \mathrm{~mL}$ $\mathrm{NaOH}(2 \mathrm{~N})$ and THF $(20 \mathrm{~mL})$. The solution was cooled to $0{ }^{\circ} \mathrm{C}$ and di-tert-butyl dicarbonate (Boc-anhydride, $\left.\mathrm{Boc}_{2} \mathrm{O}\right)(250 \mathrm{mg}, 1.1 \mathrm{mmol}$ ) was added dropwise. The resulted mixture was stirred for $4 \mathrm{~h}$ at room temperature. The reaction mixture was then concentrated in vacuo, extracted with EtOAc $(3 \times 15 \mathrm{~mL})$ and washed with $\mathrm{H}_{2} \mathrm{O}(3 \times 20 \mathrm{~mL})$. The organic phase was dried with sodium sulfate and concentrated in vacuo. The crude product was purified by column chromatography with silica gel (hexanes/EtOAc) to afford corresponding products 20-23 (Scheme 1, Figure 2)

\subsubsection{Tert-Butyl [(Z)-(hydroxyimino)(phenyl)methyl]carbamate (20)}

Compound 20 was obtained according to General method with 98\% yield (231 mg, $0.98 \mathrm{mmol})$ as white crystals; m.p. $110-111^{\circ} \mathrm{C} ;{ }^{1} \mathrm{H}$ NMR $\left(400 \mathrm{MHz}, \mathrm{CDCl}_{3}\right) \delta 7.74-7.63(\mathrm{~m}$, 2H), 7.50-7.33 (m, 3H), 5.08 (s, 2H), $1.54(\mathrm{~s}, 9 \mathrm{H}) ;{ }^{13} \mathrm{C} \mathrm{NMR}\left(100 \mathrm{MHz}, \mathrm{CDCl}_{3}\right) \delta$ 155.7, 131.1, $130.8,128.6,83.0,27.8$. Elemental analysis calculated for $\mathrm{C}_{12} \mathrm{H}_{16} \mathrm{~N}_{2} \mathrm{O}_{3}: \mathrm{C}, 61.00 \% ; \mathrm{H}, 6.83 \%$; N, $11.86 \%$; found: C, $60.95 \% ; H, 6.87 \% ; \mathrm{N}, 11.75 \%$.

\subsubsection{Tert-Butyl [(4-bromophenyl)(imino)methyl]carbamate (21)}

Compound 21 was obtained according to General method with $99 \%$ yield (248 mg, $0.99 \mathrm{mmol})$ as white crystals, m.p. $118-119{ }^{\circ} \mathrm{C} ;{ }^{1} \mathrm{H}$ NMR $\left(400 \mathrm{MHz}, \mathrm{CDCl}_{3}\right) \delta 7.62-7.45(\mathrm{~m}$, $4 \mathrm{H}), 5.08$ (s, 2H), $1.54(\mathrm{~s}, 9 \mathrm{H}) ;{ }^{13} \mathrm{C}$ NMR $\left(100 \mathrm{MHz}, \mathrm{CDCl}_{3}\right) \delta 154.8,151.9,131.8,130.0,128.1$, $125.3,83.3,27.7$. Elemental analysis calculated for $\mathrm{C}_{12} \mathrm{H}_{15} \mathrm{BrN}_{2} \mathrm{O}_{3}: \mathrm{C}, 45.73 \% ; \mathrm{H}, 4.80 \%$; $8.89 \%$; found: C, $45.83 \%$; $\mathrm{H}, 4.77 \%$;, $8.83 \%$.

\subsubsection{Tert-Butyl [N-hydroxy-2-phenylpropanimidoyl]carbamate (22)}

Compound 22 was obtained according to General method with 95\% yield (251 mg, $0.95 \mathrm{mmol})$ as white crystals; m.p. $141-142{ }^{\circ} \mathrm{C} ;{ }^{1} \mathrm{H} \mathrm{NMR}\left(400 \mathrm{MHz}, \mathrm{CDCl}_{3}\right) \delta 7.34-7.21(\mathrm{~m}$, $5 \mathrm{H}), 4.55(\mathrm{~s}, 2 \mathrm{H}), 3.73(\mathrm{q}, J=7.3 \mathrm{~Hz}, 1 \mathrm{H}), 1.53(\mathrm{~d}, J=7.3 \mathrm{~Hz}, 3 \mathrm{H}), 1.49(\mathrm{~s}, 9 \mathrm{H}) ;{ }^{13} \mathrm{C} \mathrm{NMR}$ $\left(100 \mathrm{MHz} \mathrm{CDCl}_{3}\right) \delta 159.9,152.1,140.5,128.8,127.3,41.3,27.8,17.7$. HR-MS (ESI) $(\mathrm{M}+\mathrm{H})^{+}$ $\mathrm{m} / \mathrm{z}$ calculated for $\mathrm{C}_{14} \mathrm{H}_{21} \mathrm{~N}_{2} \mathrm{O}_{3}$ : 265.1546; found: 265.1545. Elemental analysis calculated for $\mathrm{C}_{14} \mathrm{H}_{20} \mathrm{~N}_{2} \mathrm{O}_{3}$ : C, 63.62\%; H, 7.63\%; N, $10.60 \%$; found: $\mathrm{C}, 63.49 \% ; \mathrm{H}, 7.72 \% ; \mathrm{N}, 10.45 \%$.

\subsubsection{Tert-Butyl [N-hydroxy-2-phenylbutanimidoyl]carbamate (23)}

Compound 23 was obtained according to General method with 92\% yield (256 mg,

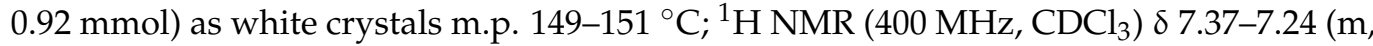
$5 \mathrm{H}), 4.55(\mathrm{~s}, 1 \mathrm{H}), 3.44(\mathrm{td}, J=7.9,0.9 \mathrm{~Hz}, 1 \mathrm{H}), 2.06-1.87(\mathrm{~m}, 2 \mathrm{H}), 1.49(\mathrm{~s}, 9 \mathrm{H}), 0.92(\mathrm{td}, J=7.3$, $0.8 \mathrm{~Hz}, 3 \mathrm{H}) ;{ }^{13} \mathrm{C} \mathrm{NMR}\left(100 \mathrm{MHz}, \mathrm{CDCl}_{3}\right) \delta 159.0,146.7,139.4,128.7,128.7,127.7,127.2,85.0$, 82.4, 48.9, 27.3, 24.7, 11.9. HR-MS (ESI) $(\mathrm{M}+\mathrm{H})^{+} \mathrm{m} / \mathrm{z}$ calculated for $\mathrm{C}_{15} \mathrm{H}_{23} \mathrm{~N}_{2} \mathrm{O}_{3}: 279.1703$; found: 279.1701 . Elemental analysis calculated for $\mathrm{C}_{15} \mathrm{H}_{22} \mathrm{~N}_{2} \mathrm{O}_{3}: \mathrm{C}, 64.73 \% ; \mathrm{H}, 7.97 \%$; , $10.06 \%$; found: C, $64.61 \% ; \mathrm{H}, 7.85 \%$; N, 9.92\%.

\subsection{General Procedure for Synthesis of Amidines 24-26}

This reaction was carried out according to the procedure described by Judkins et al., [38] to afford corresponding products 24-26 (Scheme 1, Figure 2) 


\subsubsection{Tert-Butyl [imino(phenyl)methyl]carbamate (24)}

Compound 24 was obtained according to General method with $92 \%$ yield (203 mg, $0.92 \mathrm{mmol}$ ) as white crystals; m.p. $101-102{ }^{\circ} \mathrm{C}$ (Lit. 102-103 $\left.{ }^{\circ} \mathrm{C}\right)$ [39]; ${ }^{1} \mathrm{H}$ NMR $(400 \mathrm{MHz}$, $\left.\mathrm{CDCl}_{3}\right) \delta 7.83(\mathrm{ddt}, J=7.2,3.4,1.3 \mathrm{~Hz}, 2 \mathrm{H}), 7.55-7.46(\mathrm{~m}, 1 \mathrm{H}), 7.46-7.30(\mathrm{~m}, 2 \mathrm{H}), 1.53(\mathrm{~s}, 9 \mathrm{H})$; ${ }^{13} \mathrm{C}$ NMR $\left(100 \mathrm{MHz}, \mathrm{CDCl}_{3}\right) \delta 128.5,127.1,28.1 .{ }^{1} \mathrm{H}$ and ${ }^{13} \mathrm{C}$ NMR data were in accordance with those reported in the literature [40].

\subsubsection{Tert-Butyl (2-phenylpropanimidoyl)carbamate (25)}

Compound 25 was obtained according to General method with $85 \%$ yield $(211 \mathrm{mg}$, $0.85 \mathrm{mmol})$ as white crystals; m.p. $174-176{ }^{\circ} \mathrm{C} ;{ }^{1} \mathrm{H}$ NMR $\left(400 \mathrm{MHz}, \mathrm{CDCl}_{3}\right) \delta 7.40-7.15(\mathrm{~m}$, $5 \mathrm{H}), 3.72(\mathrm{q}, J=7.3 \mathrm{~Hz}, 1 \mathrm{H}), 1.55(\mathrm{~d}, J=7.3 \mathrm{~Hz}, 3 \mathrm{H}), 1.46(\mathrm{~s}, 9 \mathrm{H}) ;{ }^{13} \mathrm{C} \mathrm{NMR}\left(100 \mathrm{MHz}, \mathrm{CDCl}_{3}\right)$ $\delta 140.1,128.9,127.8,127.6,79.5,47.0,28.1,18.6$. HR-MS (ESI) $(\mathrm{M}+\mathrm{H})^{+} \mathrm{m} / \mathrm{z}$ calculated for $\mathrm{C}_{14} \mathrm{H}_{21} \mathrm{~N}_{2} \mathrm{O}_{2}$ : 249.1597; found: 249.1595 . Elemental analysis calculated for $\mathrm{C}_{14} \mathrm{H}_{20} \mathrm{~N}_{2} \mathrm{O}_{2}$ : C, $67.72 \% ; \mathrm{H}, 8.12 \%$; N, $11.28 \%$; found: C, $67.69 \% ; \mathrm{H}, 8.02 \%$; N, $11.08 \%$.

\subsubsection{Tert-Butyl (2-phenylbutanimidoyl)carbamate (26)}

Compound 26 was obtained according to General method with $82 \%$ yield $(215 \mathrm{mg}$, $0.82 \mathrm{mmol})$ as white crystals; m.p. $164-165^{\circ} \mathrm{C} ;{ }^{1} \mathrm{H}$ NMR $\left(400 \mathrm{MHz}, \mathrm{CDCl}_{3}\right) \delta 7.41-7.22(\mathrm{~m}$, $5 \mathrm{H}), 3.45(\mathrm{dd}, J=9.5,6.0 \mathrm{~Hz}, 1 \mathrm{H}), 2.21(\mathrm{ddd}, J=13.6,7.4,6.0 \mathrm{~Hz}, 1 \mathrm{H}), 1.90(\mathrm{ddd}, J=13.8$, 9.6, 7.2 Hz, $1 \mathrm{H}), 1.48(\mathrm{~s}, 9 \mathrm{H}), 0.87(\mathrm{t}, J=7.3 \mathrm{~Hz}, 3 \mathrm{H}),{ }^{13} \mathrm{C} \mathrm{NMR}\left(100 \mathrm{MHz}, \mathrm{CDCl}_{3}\right) \delta$ 138.6, 129.0, 128.3, 127.6, 79.6, 54.9, 28.1, 26.0, 12.2. HR-MS (ESI) $(\mathrm{M}+\mathrm{H})^{+} \mathrm{m} / \mathrm{z}$ calculated for $\mathrm{C}_{15} \mathrm{H}_{23} \mathrm{~N}_{2} \mathrm{O}_{2}$ : 263.1754; found: 263.1752. Elemental analysis calculated for $\mathrm{C}_{15} \mathrm{H}_{22} \mathrm{~N}_{2} \mathrm{O}_{2}: \mathrm{C}$, $68.67 \%$; $\mathrm{H}, 8.45 \%$; N $10.68 \%$; found: $\mathrm{C}, 68.48 \%$; $\mathrm{H}, 8.39 \%$; N, $10.55 \%$.

\section{Microorganisms and Media}

The microbiological analysis procedures have been carefully described in the literature citations [41-49].

\section{Results and Discussion}

\subsection{Chemistry}

The amidoximes 1-19 (Figure 2) were chosen for another studies. For comparison, we prepared Boc-protected amidoximes 20-23 and amidines 24-26, which were obtained via reduction of parental amidoximes (Scheme 1). Amidoximes were synthesized in high yields according to general procedure consisting of addition of hydroxylamine to nitrile group in alcohol solution under reflux and sodium carbonate added as inorganic base. Furthermore, the obtained amidoximes were efficiently reduced to amidines under hydrogen atmosphere in the presence of palladium catalyst in environment of acetic acid, acetic acid anhydride and ethanol [50]. Due to the presence of free amine group in both amidines and amidoximes, they can undergo various chemical reactions of $N$-alkylation and $\mathrm{N}$-acylation. One of them is Boc-protection, often used in organic synthesis to protect amino and imino group. To date, Boc-protected amidoximes were not studied for biological activity thus it still remains unknown.

In our studies not only amidoximes and Boc-protected amidoximes were tested, but also Boc-derived amidines. Studied compounds are shown in Figure 2. The structures of all compounds were confirmed using NMR and mass spectroscopy. Melting points and spectral data of 1-26 remained in agreement with the literature data. The characterization data of the synthetized compounds $\mathbf{1 - 2 6}$ are presented in the experimental part.

\subsection{Cytotoxic Studies of the Library of Amidoxime Derivatives 1-26}

In general, the obtained results depict that the all studied amidoximes have an inhibitory effect on each bacterial model studied. Varied inhibitory activity was noted depending on the nature of the substituent in the aromatic ring of the tested compounds 1-11. Interestingly, the benzylamidoxime derivatives 12-15 exhibit lower minimal inhibitory concentration compared to the benzamidoximes 1-11. It should be noted that in the case 
of benzamidoximes 4 and 5, we observed the selectivity towards the R3 strain, while benzylamide derivative $\mathbf{1 2}$ shows selectivity and increased inhibitory activity towards the strain R2. The metoxy group introduced into aromatic ring has an unfavorable impact on the activity of benzylamidoxime 13, while it increases the activity of benzamidoxime 4 . The introduction of the Boc-group into the studied compound 20-23 reduces their inhibiting activity toward all tested strains. We noted that the removal of the hydroxyl group by reduction of the corresponding oximes is associated with a decrease in inhibitory activity of compounds 24-26. Interestingly, we have shown a great impact of the methyl group on the activity of the studied compounds. Oxime 22 exhibited higher inhibiting activity than its analogue 23, which is clearly visible on the basis of the data obtained for the analyzed strains (Figures 3-5 and Table 1).

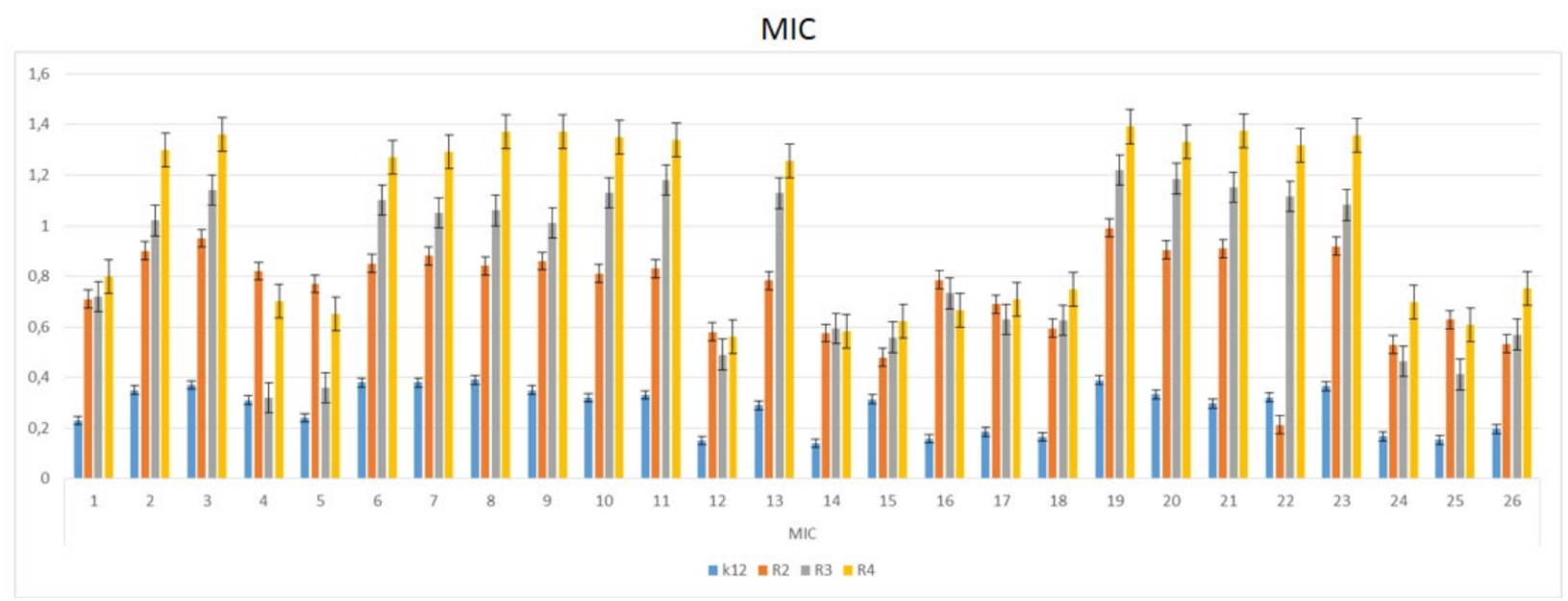

Figure 3. Minimum inhibitory concentration (MIC) of the amidoximes in model bacterial strains. The $x$-axis features amidoxime derivatives 1-26 used sequentially. The $y$-axis shows the MIC value in $\mu \mathrm{g} / \mathrm{mL}^{-1}$. Investigated strains of $E$. coli $\mathrm{K} 12$ as control (blue), R2 strains (orange), R3 strain (grey), and R4 strain (yellow). The $y$-axis shows the MBC value in $\mu \mathrm{g} / \mathrm{mL}^{-1}$. The order in which the compounds were applied to the plate are shown in Supplementary Materials Figure S1. Each experiment was performed independently in 3 replications $(n=3)$.

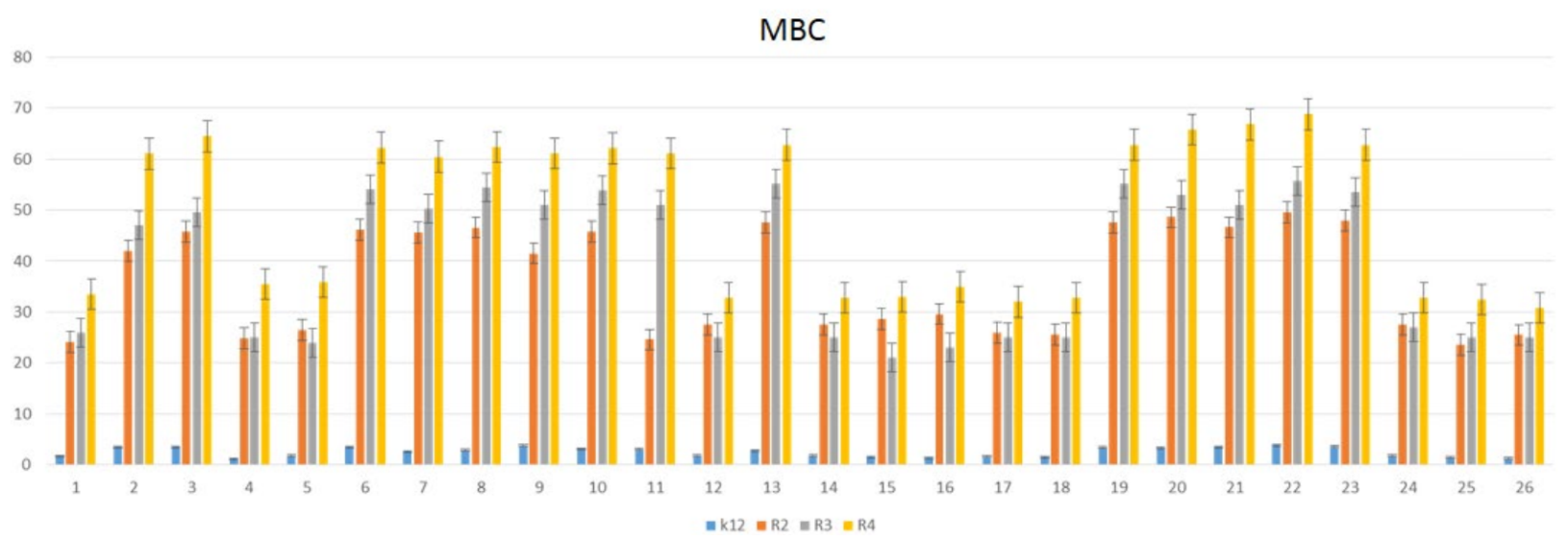

Figure 4. MBC of the amidoxime derivatives 1-26 in model bacterial strains. On the $x$-axis, 26 compounds were used sequentially. The $y$-axis shows the MBC value in $\mu \mathrm{g} / \mathrm{mL}^{-1}$ (see Supplementary Materials). Each experiment was performed independently in 3 replications $(n=3)$. 


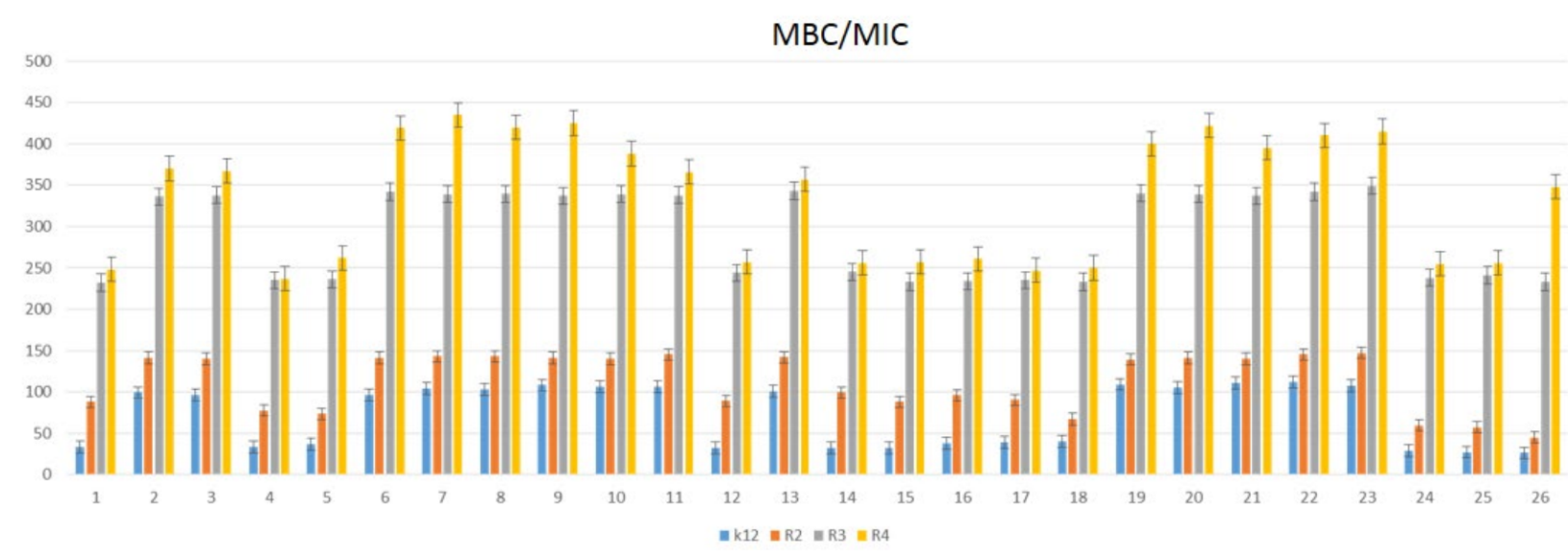

Figure 5. MBC/MIC of the amidoxime derivatives 1-26 in model bacterial strains. On the $x$-axis, compounds 1-26 are used sequentially. The $y$-axis shows the $\mathrm{MBC} / \mathrm{MIC}$ value in $\mu \mathrm{g} / \mathrm{mL}^{-1}$ (see Supplementary Materials). Each experiment was performed independently in 3 replications $(n=3)$.

Table 1. Statistical analysis of all analyzed compounds by MIC, MBC, and MBC/MIC; $<0.05^{*},<0.01^{* *},<0.001$ ***.

\begin{tabular}{|c|c|c|c|c|c|c|c|c|c|}
\hline No. of Samples & 2 & 3 & 6 & $7,8,9$ & 10,11 & 13,19 & 20,21 & 22,23 & Type of Test \\
\hline K12 & $*$ & * & $*$ & $* *$ & * & * & $* * *$ & $* * *$ & MIC \\
\hline $\mathrm{R} 2$ & $*$ & * & $*$ & $* *$ & $*$ & $*$ & $* * *$ & $* * *$ & MIC \\
\hline R3 & $*$ & * & * & $* *$ & * & * & $* * *$ & $* * *$ & MIC \\
\hline $\mathrm{R} 4$ & $*$ & * & $*$ & $* *$ & * & * & $* * *$ & $* * *$ & MIC \\
\hline K12 & $*$ & * & $* *$ & * & $* *$ & * & $* *$ & $* *$ & MBC \\
\hline $\mathrm{R} 2$ & $* *$ & * & $* *$ & * & $* *$ & * & $* *$ & $* *$ & MBC \\
\hline R3 & $* *$ & * & $* *$ & * & $* *$ & * & $* *$ & $* *$ & $\mathrm{MBC}$ \\
\hline $\mathrm{R} 4$ & $* *$ & * & $* *$ & * & $* *$ & * & $* *$ & $* *$ & MBC \\
\hline K12 & $*$ & * & $*$ & * & * & * & $* * *$ & $* * *$ & $\mathrm{MBC} / \mathrm{MIC}$ \\
\hline $\mathrm{R} 2$ & * & * & * & * & * & * & $* * *$ & $* * *$ & $\mathrm{MBC} / \mathrm{MIC}$ \\
\hline R3 & $*$ & $*$ & $*$ & $*$ & $*$ & $*$ & $* * *$ & $* * *$ & $\mathrm{MBC} / \mathrm{MIC}$ \\
\hline $\mathrm{R} 4$ & $*$ & * & $*$ & * & $*$ & * & $* * *$ & $* * *$ & $\mathrm{MBC} / \mathrm{MIC}$ \\
\hline
\end{tabular}

\subsection{The Analysis of Bacterial DNA Isolated from E. coli R2-R4 Strains Modified with Amidoxim}

Among all analyzed compounds, the highest toxicity was observed for compounds marked as 2, 3, 6, 7, 8, 9, 10, 11, 13, 19, 20, 21, 22, 23. The selected compounds used were chosen to modify model E. coli strains and, additionally, were digested with Fpg protein from the group of repair glycosylases, which is a marker of oxidative stress [41-47]. As in previous studies on various types of compounds, we also wanted to observe the effect of modification on the magnitude of oxidative damage, which should be seen as extending strands compared to unmodified forms having the three forms ccc, oc and linear. What has been observed in previous studies [41-49]. The results of bacterial DNA modified with amidoxime is shown in Figure 6 (with the action of Fpg), and in Supplementary Materials Figures S1 and S2. 


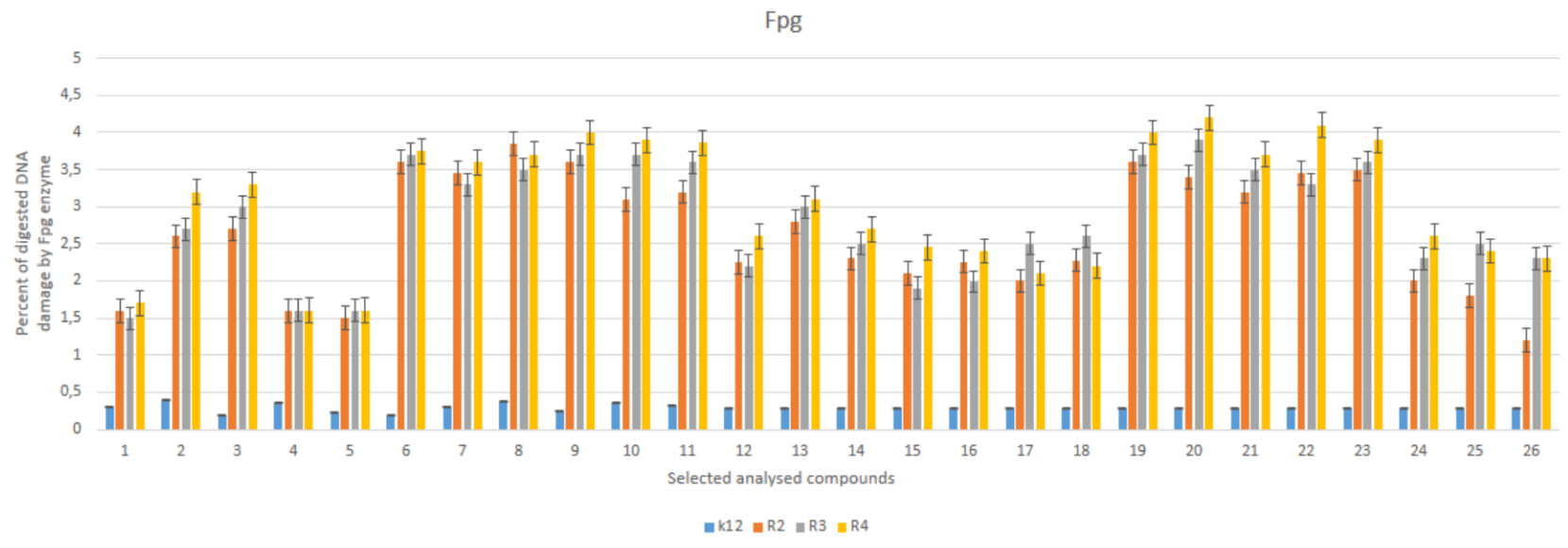

Figure 6. Percentage of plasmid DNA recognized by Fpg enzyme ( $y$-axis) with model bacterial, K12, and R2-R4 strains ( $x$-axis). The compounds numbered 2, 3, 6, 7, 8, 9, 10, 11, 13, 19, 20, 21, 22, 23 were statistically significant at $<0.05 *($ see Table 1). Each experiment was performed independently in 3 replications $(n=3)$.

The changes in the main topological forms of the plasmid: ccc, oc and linear were observed in DNA isolated from model strains and digested with Fpg protein. About $4 \%$ of oxidative damage was identified after digestion with the Fpg protein, which indicates that the analyzed compounds damage bacterial DNA very strongly due to the oxidative stress induced by them in the cell [41-49]. Our observations indicate that the length of the alkyl chain of peptidomimetics may determine the toxicity to some E. coli R4 strains, as evidenced by the MIC and MBC values [50]. The obtained results were also statistically significant at the level of $p<0.05$. (Figure 6). The obtained results indicate that the studied compounds can potentially be used as "substitutes for" commonly applied antibiotics-Figures 7 and 8 .

In the analyzed bacterial strains, after DNA isolation from them and after a modification with antibiotics and digestion with Fpg protein, no significant changes in topological forms were observed (Supplementary Materials, Figure S3). This suggests that modifications with antibiotics are less recognizable by the Fpg protein than the modifications introduced into the bacterial DNA by the analyzed compounds (Figure 8). Probably the modifications of the antibiotics used in the bacterial DNA do not cause significant changes in the topological principles caused by the specific bacterial glycosylase to which the Fpg protein belongs.

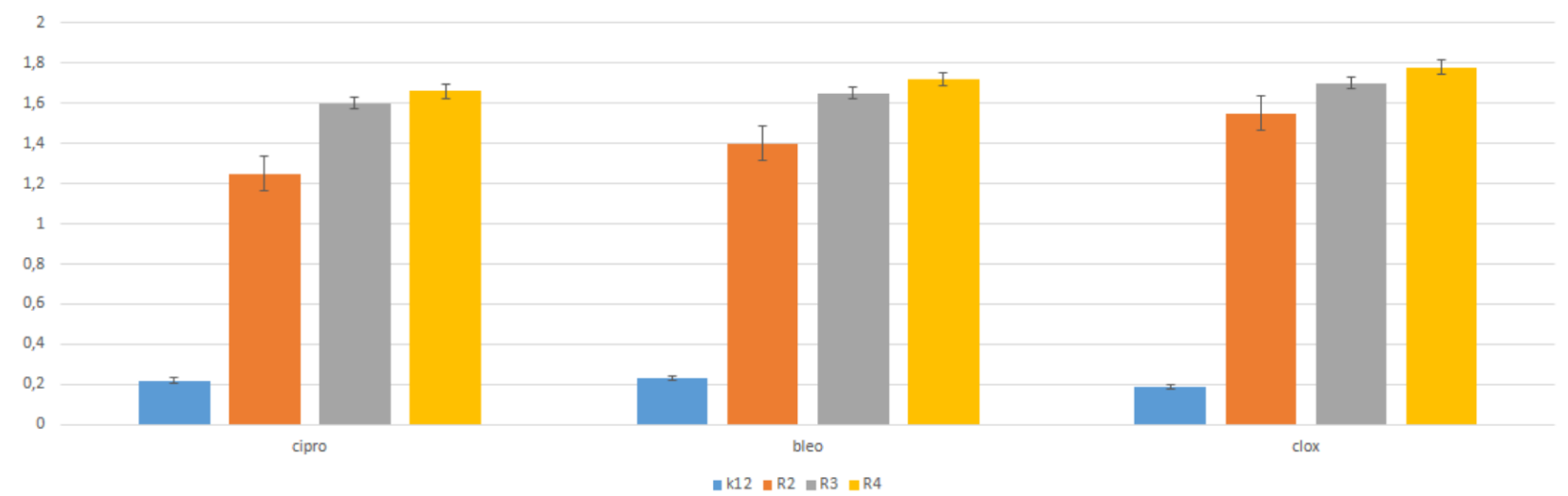

Figure 7. Examples of MIC with model bacterial strains K12, R2, R3, and R4 for studying antibiotics ciprofloxacin, bleomycin, and mechanism. The $x$-axis features antibiotics used sequentially. The $y$-axis features the MIC value in $\mu \mathrm{g} / \mathrm{mL}^{-1}$. Each experiment was performed independently in 3 replications $(n=3)$. 


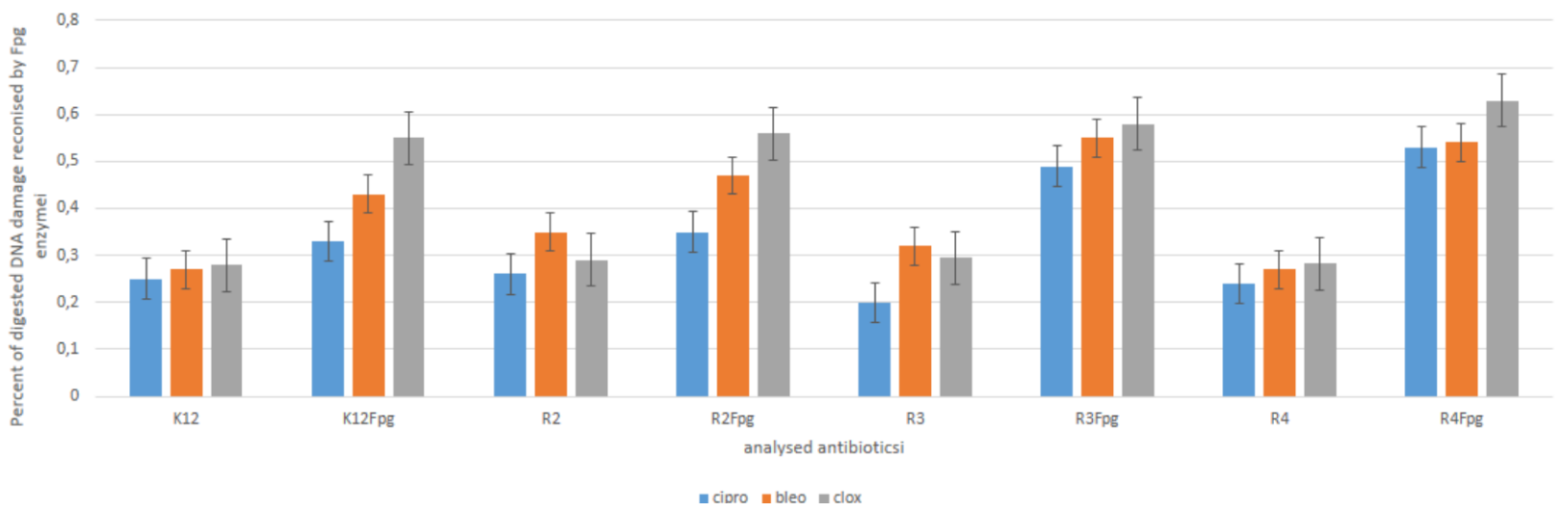

Figure 8. Percentage of bacterial DNA recognized by Fpg enzyme in model bacterial strains after ciprofloxacin, bleomycin, and cloxacillin treatment. The compounds were statistically significant at $p<0.05$. Each experiment was performed independently in 3 replications $(n=3)$.

Large modifications of plasmid DNA were observed for compounds $2,3,6,7,8,9,10$, $11,13,19,20,21,22,23$. Modifications with antibiotics were smaller and not as clear as in the case of the analyzed amidoximes. The sensitivity of E. coli strains to the cytotoxic effect of the compounds used and after Fpg protein digestion was as follows: R4 > R2 > R3 > $\mathrm{K} 12$ and this effect was very similar to our previous studies [41-49]. This indicates a very high cytotoxicity of the analyzed amidoxime derivatives towards bacterial DNA, probably resulting from the modification of the components of the bacterial membrane and the LPS contained in it, which may induce specific enzymes from the group of topoisomerases and helicases, destabilizing the structure of the exposed DNA bases. A stabilization of the complex that regulates these enzymes is perhaps necessary for cell survival. Blocking these enzymes inhibits DNA replication and rewriting, which can affect its total amount.

\section{Conclusions}

Obtained results revealed that carefully designed amidoxime derivatives may constitute a new potential source of innovative, cheap, substitutes for antibiotics against various types of bacterial microorganisms (LPS). We focused on the structure-activity relationship of compounds with an amidoximes scaffold. The obtained results showed a strong influence of the activity of all 26 compounds analyzed on the values of MIC and MBC as well as MBC/MIC for various strains of E. coli R2-R4 and K12. Based on the analysis of the above tests, 14 compounds $(2,3,6,7,8,9,10,11,13,19,20,21,22,23)$ were selected for further research. For selected compounds repair activity was compared using the Fpgglycosylase protein of the BER pathway (base excision repair), which is consistent with our research hypothesis. The above results are very important for research on the mechanism of cytotoxic action of new compounds as innovative and safe drugs based on amidoxime derivatives, which may lead to the destruction of the bacterial cell membrane by changing its surface charge and may play a significant role in changing its electrokinetic potential expressing with the reversal of loads. A special effect was observed for compounds 2, 3, $6,7,8,9,10,11,13,19,20,21,22,23$, which showed certain MIC values and MBC/MIC ratios. Compounds no. 7 and 21 showed super-selectivity in all analyzed bacterial strains, even differentiating the cytotoxicity in the K12 strain. It should be noted that in the case of benzamidoximes 4 and 5, the selectivity towards the R3 strain was observed, while benzylamide derivative 12 shows selectivity and increased inhibitory activity towards the strain R2. The reported compounds may be highly specific for pathogenic E. coli strains on the basis of the model strains used. In the future, cytotoxicity studies will also be conducted using various cell lines and cultures to assess the biocompatibility of test compounds for active peptidomimetics 
Supplementary Materials: The following are available online at https: / www.mdpi.com/article/ 10.3390/ma14247577/s1. Figure S1: Examples of MIC and MBC on microplates with different concentration of studied compounds $\left(\mathrm{mg} \mathrm{L}^{-1}\right)$. Figure S2: Example of an agarose gel electrophoresis separation of isolated plasmids DNA from R4 strains modified with selected coumarin derivatives. Figure S3: Example of an agarose gel electrophoresis separation of isolated plasmids DNA from R4 strains modified with antibiotics: cloxacillin, ciprofloxacin, and bleomycin digested (or not) with repair enzymes Fpg. ${ }^{1} \mathrm{H}$ and ${ }^{13} \mathrm{C}$ NMR spectra of compounds 1-26.

Author Contributions: Conceptualization or design of the work, P.K., D.K. and R.O.; methodology, P.K., J.S.-G., A.B., R.O., M.S., K.K., synthesis of all compounds, J.S.-G., D.K.; software, P.K. and M.S.; validation, P.K., J.S.-G., D.K., R.O., M.S., K.K., formal analysis, P.K., J.S.-G., D.K. and R.O.; investigation, P.K., M.S., interpretation of data for the work, P.K. and D.K.; drafting the work, P.K. and D.K.; revising it critically for important intellectual content; P.K., D.K. and K.K. resources; P.K., R.O., K.K., data curation, P.K. and D.K.; writing of the original draft preparation, P.K. and D.K.; writing of review and editing, P.K.; visualization, P.K. and M.S.; supervision, P.K. and D.K.; project administration, P.K. and R.O.; funding acquisition, K.K. All authors have read and agreed to the published version of the manuscript.

Funding: This work was supported by a grant from the Medical University of Białystok SUB/2DN/ $21 / 001 / 2201$ and SUB/2/DN/20/003/2201 and by the National Science Center, Poland, project OPUS No. 2019/33/B/ST4/01118.

Institutional Review Board Statement: Not applicable.

Informed Consent Statement: Not applicable.

Data Availability Statement: Upon request of those interested.

Acknowledgments: The authors thank Jolanta Łukasiewicz from Ludwik Hirszfeld Institute of Immunology and Experimental Therapy (Polish Academy of Sciences) for providing the strains of E. coli. We would also like to thank Dominik Koszelewski for his contribution to the analysis of the obtained data.

Conflicts of Interest: The authors declare no conflict of interest.

\author{
Abbreviations \\ MIC minimum inhibitory concentration \\ MBC minimum bactericidal concentration \\ Oc open circle \\ Ccc covalently closed circle \\ BER base excision repair \\ Fpg DNA-formamidopyrimidine glycosylase
}

\title{
References
}

1. Sahyoun, T.; Arrault, A.; Schneider, R. Amidoximes and Oximes: Synthesis, Structure, and Their Key Role as NO Donors. Molecules 2019, 24, 2470. [CrossRef]

2. Jousserandot, A.; Boucher, J.L.; Henry, Y.; Niklaus, B.; Clement, B.; Mansuy, D. Microsomal cytochrome P450 dependent oxidation of $N$-hydroxyguanidines, amidoximes, and ketoximes: Mechanism of the oxidative cleavage of their $\mathrm{C}=\mathrm{N}(\mathrm{OH})$ bond with formation of nitrogen oxides. Biochemistry 1998, 37, 17179-19191. [CrossRef] [PubMed]

3. Logan, R.T.; Redpath, J.; Roy, R.G. Indene and Naphthalene Derivatives. Patent E.P. 0,199,393, 29 October 1986.

4. Kliachyna, M.; Santoni, G.; Nussbaum, V.; Renou, J.; Sanson, B.; Colletier, J.P.; Arboléas, M.; Loiodice, M.; Weik, M.; Jean, L.; et al. Design, synthesis and biological evaluation of novel tetrahydroacridine pyridine- aldoxime and -amidoxime hybrids as efficient uncharged reactivators of nerve agent-inhibited human acetylcholinesterase. Eur. J. Med. Chem. 2014, 78, 455-467. [CrossRef] [PubMed]

5. Hazeldine, S.; Pachaiyappan, B.; Steinbergs, N.; Nowotarski, S.; Hanson, A.S.; Casero, R.A., Jr.; Woster, P.M. Low Molecular Weight Amidoximes that Act as Potent Inhibitors of Lysine-Specific Demethylase 1. J. Med. Chem. 2012, 55, 7378-7391. [CrossRef]

6. Ganem, B.; Dong, Y.; Zheng, Y.F.; Prestwich, G.D. Amidrazone and Amidoxime Inhibitors of Squalene Hopene Cyclase. J. Org. Chem. 1999, 64, 5441-5446. [CrossRef] [PubMed]

7. Sirelkhatim, N.; LaJeunesse, D.; Kelkar, A.D.; Zhang, L. Antifungal activity of amidoxime surface functionalized electrospun polyacrylonitrile nanofibers. Mater. Lett. 2015, 141, 217-220. [CrossRef] 
8. Hea, N.; Lia, H.; Chenga, C.; Donga, H.; Lub, X.; Wena, J.; Wang, X. Enhanced marine applicability of adsorbent for uranium via synergy of hyperbranched poly(amido amine) and amidoxime groups. Chem. Engin. J. 2020, 395, 125162. [CrossRef]

9. Abele, E.; Abele, R.; Lukevics, E. Indole and Isatin Oximes: Synthesis, Reactions and Biological Activity. Chem. Heterocycl. Comp. 2003, 39, 3-35. [CrossRef]

10. Ansede, J.H.; Anbazhagan, M.; Brun, R.; Easterbrook, J.D.; Edwin Hall, J.; Boykin, D.W. O-Alkoxyamidine Prodrugs of Furamidine: In Vitro Transport and Microsomal Metabolism as Indicators of in Vivo Efficacy in a Mouse Model of Trypanosoma brucei rhodesiense Infection. J. Med. Chem. 2004, 47, 4335-4338. [CrossRef]

11. Kotthaus, J.; Steinmetzer, T.; van de Locht, A.; Clement, B. Analysis of highly potent amidine containing inhibitors of serine proteases and their N-hydroxylated prodrugs (amidoximes). J. Enzym. Inhib. Med. Chem. 2011, 26, 115-122. [CrossRef] [PubMed]

12. Shigeno, M.; Nakaji, K.; Nozawa-Kumada, K.; Kondo, Y. Catalytic Amide-Base System of TMAF and N(TMS) 3 for Deprotonative Coupling of Benzylic C(sp3)-H Bonds with Carbonyls. Org. Lett. 2019, 21, 2588-2592. [CrossRef] [PubMed]

13. Zhang, Y.-G.; Liu, X.-L.; He, Z.-Y.; Li, X.-M.; Kang, H.-J.; Tian, S.-K. Palladium/Copper-Catalyzed Oxidative Arylation of Terminal Alkenes with Aroyl Hydrazides. Chem. Eur. J. 2014, 20, 2765-2769. [CrossRef] [PubMed]

14. Chen, L.; Lang, H.; Fang, L.; Yu, J.; Wang, L. Nickel-Catalyzed Desulfitative Suzuki-Miyaura Cross-Coupling of N,NDisulfonylmethylamines and Arylboronic Acids. Eur. J. Org. Chem. 2014, 2014, 6385-6389. [CrossRef]

15. Shen, T.; Wang, T.; Qin, C.; Jiao, N. Silver-Catalyzed Nitrogenation of Alkynes: A Direct Approach to Nitriles through C=C Bond Cleavage. Angew. Chem. Int. Ed. 2013, 52, 6677-6680. [CrossRef] [PubMed]

16. Baykov, S.V.; Kotlyarova, V.D.; Shetnev, A.A.; Tarasenko, M.V. 2-(1,2,4-Oxadiazol-5-yl)anilines Based on Amidoximes and Isatoic Anhydrides: Synthesis and Structure Features. Russ. J. Gen. Chem. 2021, 91, 768-778. [CrossRef]

17. Kozlov, M.V.; Kleymenova, A.A.; Romanova, L.I.; Konduktorov, K.A.; Smirnova, O.A.; Prasolov, V.S.; Kochetkova, S.N. Benzohydroxamic acids as potent and selective anti-HCV agents. Bioorg. Med. Chem. Lett. 2013, 23, 5936-5940. [CrossRef] [PubMed]

18. Tarasenko, M.; Duderin, N.; Sharonova, T.; Baykov, S.; Shetnev, A.; Smirnov, A.V. Room-temperature synthesis of pharmaceutically important carboxylic acids bearing the 1,2,4-oxadiazole moiety. Tetrahedron Lett. 2017, 58, 3672-3677. [CrossRef]

19. Cai, Y.; Qian, X.; Rérat, A.; Auffrant, A.; Gosmini, C. Cobalt-Catalyzed Electrophilic Cyanation of Arylzinc Halides with N-Cyano-N-phenyl-p-methylbenzenesulfonamide (NCTS) Adv. Synth. Catal. 2015, 357, 3419-3423. [CrossRef]

20. Kumpan, K.; Nathubhai, A.; Zhanga, C.; Wood, P.J.; Lloyd, M.D.; Thompson, A.S.; Haikarainen, T.; Lehtiö, L.; Threadgill, M.D. Structure-based design, synthesis and evaluation in vitro of arylnaphthyridinones, arylpyridopyrimidinones and their tetrahydro derivatives as inhibitors of the tankyrases. Bioorg. Med. Chem. 2015, 23, 3013-3032. [CrossRef]

21. Presnukhina, S.; Tarasenko, M.; Baykov, S.; Smirnov, S.N.; Boyarskiy, V.P.; Shetnev, A.; Korsakov, M.K. Entry into (E)-3-(1,2,4oxadiazol-5-yl)acrylic acids via a one-pot ring-opening/ring-closing/retro-Diels-Alder reaction sequence. Tetrahedron Lett. 2020, 61, 151543. [CrossRef]

22. Lin, C.C.; Hsieh, T.H.; Liao, P.Y.; Liao, Z.Y.; Chang, C.W.; Shih, Y.C.; Yeh, W.H.; Chien, T.C. Practical Synthesis of N-Substituted Cyanamides via Tiemann Rearrangement of Amidoximes. Org. Lett. 2014, 16, 892-895. [CrossRef] [PubMed]

23. Saint-André, G.; Kliachyna, M.; Kodepelly, S.; Louise-Leriche, L.; Gillon, E.; Renard, P.Y.; Nachon, F.; Baati, R.; Wagner, A. Design, synthesis and evaluation of new $\alpha$-nucleophiles for the hydrolysis of organophosphorus nerve agents: Application to the reactivation of phosphorylated acetylcholinesterase. Tetrahedron 2011, 67, 6352-6361. [CrossRef]

24. Hiroto, T. Oxadiazole Compound and Use Thereof as Pesticides. Patent WO 2018/30460, 2018. (A1).

25. Partridge, M.W. Derivatives of Diphenyl Ether as Antituberculous Compounds. J. Pharm. Pharmacol. 1952, 4, 533-538. [CrossRef] [PubMed]

26. Shabalin, D.A.; Dunsford, J.J.; Ngwerume, S.; Saunders, A.R.; Gill, D.M.; Camp, J.E. Synthesis of 2,4-Disubstituted Imidazoles via Nucleophilic Catalysis. Synlett 2020, 31, 797-800. [CrossRef]

27. Conole, D.; Beck, T.M.; Jay-Smith, M.; Tingle, M.D.; Eason, C.T.; Brimble, M.A.; Rennison, D. Synthesis and methemoglobinemiainducing properties of benzocaine isosteres designed as humane rodenticides. Bioorg. Med. Chem. 2014, 22, 2220-2235. [CrossRef] [PubMed]

28. Dong, C.Z.; Ahamada-Himidi, A.; Plocki, S.; Aoun, D.; Touaibia, M.; Meddad-Bel Habich, N.; Huet, J.; Redeuilh, C.; Ombetta J.E.; Godfroid, J.J.; et al. Inhibition of secretory phospholipase A2. 2-Synthesis and structure-activity relationship studies of 4,5-dihydro-3-(4-tetradecyloxybenzyl)-1,2,4-4H-oxadiazol-5-one (PMS1062) derivatives specific for group II enzyme. Bioorg. Med. Chem. 2005, 13, 1989-2007. [CrossRef] [PubMed]

29. Tsyganov, D.V.; Khrustalev, V.N.; Konyushkin, L.D.; Raihstat, M.M.; Firgang, S.I.; Semenov, R.V.; Kiselyov, A.S.; Semenova, M.N.; Semenov, V.V. 3-(5-)-Amino-o-diarylisoxazoles: Regioselective synthesis and antitubulin activity. Eur. J. Med. Chem. 2014, 73, 112-125. [CrossRef]

30. Geyl, K.; Baykov, S.; Tarasenko, M.; Zelenkov, L.E.; Matveevskaya, V.; Boyarskiy, V.P. Convenient entry to N-pyridinylureas with pharmaceutically privileged oxadiazole substituents via the acid-catalyzed C-H activation of N-oxides. Tetrahedron Lett. 2019, 60, 151108. [CrossRef]

31. Tarasenko, M.V.; Presnukhina, S.I.; Baikov, S.V.; Shetnev, A.A. Synthesis and Evaluation of Antibacterial Activity of 1,2,4Oxadiazole-Containing Biphenylcarboxylic Acids. Russ. J. Gen. Chem. 2020, 90, 1611-1619. [CrossRef] 
32. Yang, X.; Liu, G.; Li, H.; Zhang, Y.; Song, D.; Li, C.; Wang, R.; Liu, B.; Liang, W.; Jing, Y.; et al. Novel Oxadiazole Analogues Derived from Ethacrynic Acid: Design, Synthesis, and Structure-Activity Relationships in Inhibiting the Activity of Glutathione S-Transferase P1-1 and Cancer Cell Proliferation. J. Med. Chem. 2010, 53, 1015-1022. [CrossRef] [PubMed]

33. Li, Y.; Zhu, H.; Chen, K.; Liu, R.; Khallaf, A.; Zhang, X.; Ni, J. Synthesis, insecticidal activity, and structure-activity relationship (SAR) of anthranilic diamides analogs containing oxadiazole rings. Org. Biomol. Chem. 2013, 11, 3979-3988. [CrossRef]

34. Freund, M.; Lenze, F. Ueber ein Polymerisationsproduct des Trimethylacetonitrils. Chem. Ber. 1891, 24, 2161-21671. [CrossRef]

35. Yu, S.; Sanchez, T.W.; Liu, Y.; Yin, Y.; Neamati, N.; Zhao, G. Design and synthesis of novel pyrimidone analogues as HIV-1 integrase inhibitors. Bioorg. Med. Chem. Lett. 2013, 23, 6134-6137. [CrossRef] [PubMed]

36. Lee, W.M. Stabilization of Hydroxylamine Containing Solutions and Methods for Their Preparation. Patent US2009/112024, 7 April 2009. (A1).

37. Detistov, O.S.; Orlov, V.D.; Zhuravel', I.O. Isomeric 3-Isoxadiazolylcoumarins and Their Derivatives. J. Heterocycl. Chem. 2012, 49, 883-892. [CrossRef]

38. Judkins, B.D.; Allen, D.G.; Cook, T.A.; Evans, B.; Sardharwala, T.E. A Versatile Synthesis of Amidines from Nitriles Via Amidoximes. Synth. Comm. 1998, 26, 4351-4367. [CrossRef]

39. Guzmán, A.; Romero, M.; Talamás, F.X.; Villena, R.; Greenhouse, R.; Muchowski, J.M. 1,3-Diaza-1,3-butadienes. Synthesis and Conversion into Pyrimidines by $[4 \pi+2 \pi]$ Cycloaddition with Electron Deficient Acetylenes. Synthetic Utility of 2-(Trichloromethyl)pyrimidines. J. Org. Chem. 1996, 61, 2470-2483. [CrossRef]

40. Baeten, M.; Maes, B.U.W. Guanidine Synthesis: Use of Amidines as Guanylating Agents. Adv. Synth. Catal. 2016, 358, 826-833. [CrossRef]

41. Kowalczyk, P.; Trzepizur, D.; Szymczak, M.; Skiba, G.; Kramkowski, K.; Ostaszewski, R. 1,2-Diarylethanols-A New Class of Compounds That Are Toxic to E. coli K12, R2-R4 Strains. Materials 2021, 14, 1025. [CrossRef] [PubMed]

42. Kowalczyk, P.; Madej, A.; Szymczak, M.; Ostaszewski, R. $\alpha$-Amidoamids as New Replacements of Antibiotics-Research on the Chosen K12, R2-R4 E. coli Strains. Materials 2020, 13, 5169. [CrossRef] [PubMed]

43. Kowalczyk, P.; Borkowski, A.; Czerwonka, G.; Cłapa, T.; Cieśla, J.; Misiewicz, A.; Borowiec, M.; Szala, M. The microbial toxicity of quaternary ammonium ionic liquids is dependent on the type of lipopolysaccharide. J. Mol. Liq. 2018, 266, 540-547. [CrossRef]

44. Borkowski, A.; Kowalczyk, P.; Czerwonka, G.; Cieśla, J.; Cłapa, T.; Misiewicz, A.; Szala, M.; Drabik, M. Interaction of quaternary ammonium ionic liquids with bacterial membranes-Studies with Escherichia coli R1-R4-type lipopolysaccharides. J. Mol. Liq. 2017, 246, 282-289. [CrossRef]

45. Kowalczyk, P.; Gawdzik, B.; Trzepizur, D.; Szymczak, M.; Skiba, G.; Raj, S.; Kramkowski, K.; Lizut, R.; Ostaszewski, R. $\delta$ Lactones-A New Class of Compounds That Are Toxic to E. coli K12 and R2-R4 Strains. Materials 2021, 14, 2956. [CrossRef] [PubMed]

46. Dissanayake, D.R.; Wijewardana, T.G.; Gunawardena, G.A.; Poxton, I.R. Distribution of lipopolysaccharide core types among avian pathogenic Escherichia coli in relation to the major phylogenetic groups. Vet. Microbiol. 2008, 132, 355-363. [CrossRef]

47. Maciejewska, A.; Kaszowska, M.; Jachymek, W.; Lugowski, C.; Lukasiewicz, J. Lipopolysaccharide-linked Enterobacterial Common Antigen (ECALPS) Occurs in Rough Strains of Escherichia coli R1, R2, and R4. Int. J. Mol. Sci. 2020, 21, 6038. [CrossRef] [PubMed]

48. Prost, M.E.; Prost, R. Basic parameters of evaluation of the effectiveness of antibiotic therapy. OphthaTherapy 2017, 4, 233-236. [CrossRef]

49. Kowalczyk, P.; Wilk, M.; Parul, P.; Szymczak, M.; Kramkowski, K.; Raj, S.; Skiba, G.; Sulejczak, D.; Kleczkowska, P.; Ostaszewski, R. The Synthesis and Evaluation of Aminocoumarin Peptidomimetics as Cytotoxic Agents on Model Bacterial E. coli Strains. Materials 2021, 14, 5725. [CrossRef] [PubMed]

50. Batt, D.G.; Houghton, G.C.; Daneker, W.F.; Jadhav, P.K. Synthesis of Cis and Trans Isomers of an Isoxazoline Ring-Hydroxylated Metabolite of Roxifiban, a Platelet Glycoprotein IIb/IIIa Receptor Antagonist. J. Org. Chem. 2000, 65, 8100-8104. [CrossRef] [PubMed] 